\title{
Ultrasound Vector Flow Imaging: Part I: Sequential Systems
}

\author{
Jensen, Jørgen Arendt; Nikolov, Svetoslav Ivanov; Yu, Alfred C. H.; Garcia, Damien
}

Published in:

IEEE Transactions on Ultrasonics, Ferroelectrics and Frequency Control

Link to article, DOI:

10.1109/TUFFC.2016.2600763

Publication date:

2016

Document Version

Peer reviewed version

Link back to DTU Orbit

Citation (APA):

Jensen, J. A., Nikolov, S. I., Yu, A. C. H., \& Garcia, D. (2016). Ultrasound Vector Flow Imaging: Part I:

Sequential Systems. IEEE Transactions on Ultrasonics, Ferroelectrics and Frequency Control, 63(11), 1704 1721. https://doi.org/10.1109/TUFFC.2016.2600763

\section{General rights}

Copyright and moral rights for the publications made accessible in the public portal are retained by the authors and/or other copyright owners and it is a condition of accessing publications that users recognise and abide by the legal requirements associated with these rights.

- Users may download and print one copy of any publication from the public portal for the purpose of private study or research.

- You may not further distribute the material or use it for any profit-making activity or commercial gain

- You may freely distribute the URL identifying the publication in the public portal 


\title{
Ultrasound Vector Flow Imaging: I: Sequential Systems
}

\author{
Jørgen Arendt Jensen ${ }^{1}$, Fellow, IEEE, Svetoslav Ivanov Nikolov ${ }^{2}$, Alfred C. H. Yu ${ }^{3}$ and Damien Garcia ${ }^{4}$ \\ ${ }^{1}$ Center for Fast Ultrasound Imaging, Department of Electrical Engineering, \\ Technical University of Denmark, DK-2800 Lyngby, Denmark \\ ${ }^{2}$ BK Ultrasound, Mileparken, Herlev, Denmark \\ ${ }^{3}$ Department of Electrical and Computer Engineering, University of Waterloo, Waterloo, ON, Canada \\ ${ }^{4}$ Research Unit of Biomechanics \& Imaging in Cardiology, \\ University of Montreal Hospital, Quebec, Canada
}

\begin{abstract}
The paper gives a review of the most important methods for blood velocity vector flow imaging (VFI) for conventional, sequential data acquisition. This includes multibeam methods, speckle tracking, transverse oscillation, color flow mapping derived vector flow imaging, directional beamforming, and variants of these. The review covers both 2-D and 3-D velocity estimation and gives a historical perspective on the development along with a summary of various vector flow visualization algorithms. The current state-of-the-art is explained along with an overview of clinical studies conducted and methods for presenting and using VFI. A number of examples of VFI images are presented, and the current limitations and potential solutions are discussed.
\end{abstract}

\section{INTRODUCTION}

This two part paper gives a review of vector flow imaging (VFI) in medical ultrasound with a broad overview of the developed methods, their function and use. The review is divided into this Part I covering imaging systems with sequential data acquisition, where a focused field is emitted in one direction and received beamforming is performed before estimating the velocity vector along this direction. The accompanying Part II paper describes systems with parallel data acquisition, where the ultrasound field is emitted broadly as e.g. a spherical or plane wave [1].

It was early recognized that conventional velocity estimation systems or Doppler systems only could estimate the velocity component along the ultrasound beam. The frequency $f_{p}$ of the returned signal for pulse-wave systems is given by [2], [3], [4]:

$$
f_{p}=\frac{2|\vec{v}| \cos \theta}{c} f_{0}=\frac{2 v_{z}}{c} f_{0}=\frac{2 v_{z}}{\lambda},
$$

where $f_{0}$ is the emitted ultrasound frequency, $\vec{v}$ is the blood velocity, $\lambda=c / f_{0}$ is the wavelength, and $c$ is the speed of sound. The beam-to-flow angle is $\theta$ and the axial velocity component is, thus, $v_{z}=|\vec{v}| \cos \theta$. The angle should be kept below $60^{\circ}$ to maintain an accurate estimate [4], [5], which is often difficult in a clinical setting, either because the vessel is perpendicular to the ultrasound beam or because the angle varies over the cardiac cycle.

This early on led to the suggestion of methods for finding the velocity components in 2 or 3 directions. The first ap- proaches [6] used two crossing beams as described in Section II-A. A second approach suggested by Trahey et al. [7] to track the speckle pattern between frames is described in Section III. The conventional axial estimation relies on the ultrasound oscillation to find either the frequency or phase shift. Introducing a lateral oscillation as suggested by Jensen, Munk and Anderson [8], [9] makes it possible to find the lateral velocity component as described in Section IV. A number of other approaches like directional beamforming, color Dopplerderived vector flow mapping, estimation of bandwidth, and spectral alignment have also been suggested and are detailed in Section V.

The methods mentioned above were primarily introduced for 2-D velocity estimation, but they can also be translated to full 3-D methods. This is elucidated in Section VIII. The display of vector flow information is a challenge, since the human circulation houses complex and pulsating flow. Vortices, disturbed, and turbulent flow are present and appear in very short time intervals during the cardiac cycle. Efforts to handle this are described in Section IX.

The first commercial implementations of the different methods are starting to appear in clinical scanners and along with experimental research systems has made it possible to conduct the first clinical studies of VFI. An overview of the these studies and implications are given in Section $X$. The paper is concluded with a discussion of the benefits and future possibilities of VFI in Section XI.

\section{Multi-Beam Doppler Estimation Methods}

\section{A. General Principles}

The use of multiple beams from different angles to derive flow vector information can be considered as one of the earliest approaches developed in the field [10], [11]. Commonly referred to as cross-beam Doppler or multi-beam Doppler [12], this approach is founded upon a generalized form of (1), which states that the frequency shift of echoes returned from a moving blood scatterer is given by:

$$
f_{p}=\frac{|\vec{v}|\left(\cos \theta_{T}-\alpha\right)+|\vec{v}|\left(\cos \varphi_{R}-\alpha\right)}{c} f_{0},
$$




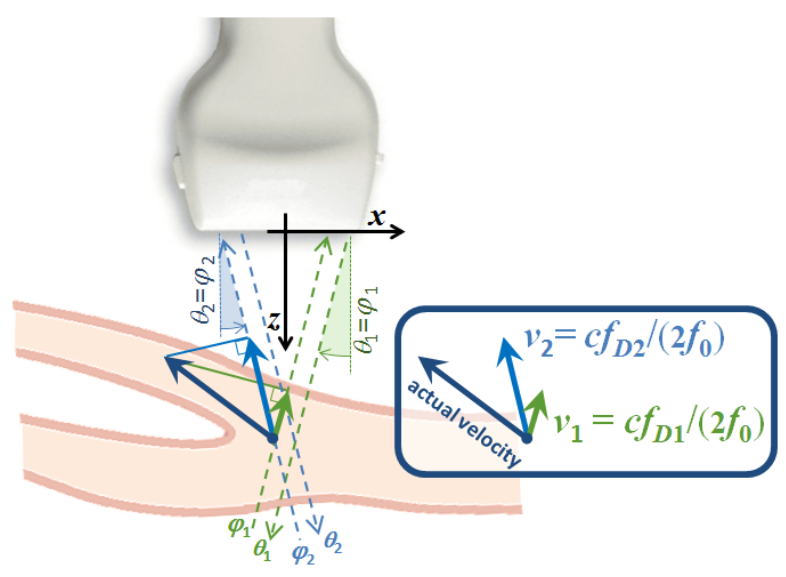

Fig. 1. Illustration of cross-beam vector flow acquisition. This figure represents a special case of the multi-beam scheme with $M N=2$ and $\left(\theta_{1} ; \theta_{2}\right)=\left(\phi_{1} ; \phi_{2}\right)$.

where $|\vec{v}|$ and $\alpha$ respectively denote the velocity magnitude and angle that the blood scatterer is moving at (both of which are unknown); $\theta_{T}$ and $\varphi_{R}$ are the transmit and receive beam angles.

The generalized form of the frequency shift equation given in (2) can be readily rewritten in terms of axial velocity $v_{z}=$ $|\vec{v}| \cos \alpha$ and lateral velocity $v_{x}=|\vec{v}| \sin \alpha$ by substituting the trigonometry relation $\cos A-B=\cos A \cos B+\sin A \sin B$ into (2) such that it becomes:

$$
v_{z}\left(\cos \theta_{T}+\cos \varphi_{R}\right)+v_{x}\left(\sin \theta_{T}+\sin \varphi_{R}\right)=\frac{c}{f_{0}} f_{p} .
$$

As known from linear algebra, $v_{z}$ and $v_{x}$ (i.e. the constituent components of the flow vector) can be found by: (i) measuring the Doppler frequency from different beam-flow angles, and (ii) constructing a system of frequency shift equations to solve for the individual flow vector components. A minimum of two frequency shift measurements need to be made to obtain two independent realizations of frequency shift equations. An example is shown in Fig. 1, where two pairs of identical transmit and receive beam angles are used [i.e. $\left(\theta_{1}, \theta_{2}\right)=\left(\varphi_{1}, \varphi_{2}\right)$ ].

\section{B. Dual-Beam Implementations}

From a matrix formulation standpoint, with two frequency shift measurements, $v_{z}$ and $v_{x}$ in (3) can be solved as a matrix inverse problem by forming a linear system of two equations with two unknowns. Centered around this principle, different versions of dual-beam Doppler flow vector estimation schemes have been proposed over the years [12]. Early formulations, intended merely for single-gate flow vector measurements [10], [13], [14], involve the use of two separate transducers (left and right) that are slanted toward each other such that they have an overlapping beam region. During operation, the left and right transducers would alternate as the transmitter and receiver in a sequence of pulse firing events, so that the two transmit-receive angle pairs are essentially trigonometric complements. Variations of this scheme exist [11], [15], and some of them involved hybrid use of Doppler spectral broadening principles to aid the flow vector determination process [16], [17]. The downside of this approach is that the effective
PRF is inevitably halved, as each flow vector must be derived from two separate pulse firing events.

As array transducer technology matured, splitting the aperture into two halves became feasible [18], [19]. Also, deriving flow vectors over an entire field of view can be achieved [20], [21]. For this split aperture approach, a transmit pulse is fired axially from the center of the array aperture without beam steering, and two receive apertures are formed from the left and right sides of the array transducer [18], [22]. The benefit of using this approach is that one pulse firing event is sufficient to derive a flow vector, which may be computed from specialcase formulas involving the difference and sum of Doppler frequencies detected from the two receive sub-apertures [23]. However, these formulations have not seen widespread clinical use likely because of the technical difficulty in reprogramming clinical scanners that are often designed using an embedded system design approach.

The performance of dual-beam Doppler flow vector estimation schemes have been characterized in detail [24], [25], [26], [27]. Although, comparing with speckle tracking methods, dual-beam Doppler flow vector estimation methods are more robust in cases where blood flow partially goes out of the imaging plane such that the measured flow vector is merely a planar projection of the true 3D flow vector [28], [29], it suffers from one critical weakness: that is, any errors in one of the two frequency shift measurements would give rise to significant vector estimation variance. This issue can be overcome by extending the principles to a multi-beam implementation, as will be discussed in the next subsection.

\section{Multi-Beam Implementations}

Instead of only obtaining two realizations of frequency shift measurements for flow vector estimation purpose, it is possible to generalize the framework to incorporate more than two transmit-receive angle pairs [30], [31]. The benefit of such a multi-beam implementation is that the flow vector estimation problem, which is a matrix inverse problem, can be re-formulated as an over-determined system of equations that carries the following matrix form [32]:

$\left[\begin{array}{cc}\cos \theta_{1}+\cos \varphi_{1} & \sin \theta_{1}+\sin \varphi_{1} \\ \cos \theta_{2}+\cos \varphi_{2} & \sin \theta_{2}+\sin \varphi_{2} \\ \vdots & \vdots \\ \cos \theta_{M}+\cos \varphi_{N} & \sin \theta_{M}+\sin \varphi_{N}\end{array}\right]\left[\begin{array}{c}v_{z} \\ v_{x}\end{array}\right]=\frac{c_{o}}{f_{0}}\left[\begin{array}{c}f_{p 1} \\ f_{p 2} \\ \vdots \\ f_{p(M N)}\end{array}\right]$.

With $M N$ realizations of frequency shift measurements, the flow vector computation process essentially becomes a leastsquares fitting problem that would yield estimates with minimum mean squared error [32], [33]. Using the matrix notation from (4) as $\mathbf{A v}=\mathbf{u}$, the least-squares estimation approach is solved for the flow vector $\mathbf{v}$ through the pseudo-inverse operation:

$$
\mathbf{v}=\left(\mathbf{A}^{T} \mathbf{A}\right)^{-1} \mathbf{A}^{T} \mathbf{u} .
$$

Note that calculation of the pseudo-inverse is after all required, since $\mathbf{A}$ is a $M N \times 2$ non-square matrix.

Although the multi-beam Doppler vector estimation scheme appears to be more algebraically intensive, its realization is 
in practice rather straightforward. The reason is because the pseudo-inverse is merely a constant matrix quantity that can be pre-computed and applied to every instance of least-squares flow vector estimation. As shown in [33], with the use of highperformance computing hardware such as graphics processing units [34], [35], flow vectors can be derived consistently at all flow sample volume positions in the field of view at real-time processing throughputs.

\section{SPECKLE TRACKING AND PARTICLE VELOCITY} IMAGING

Ultrasound vector flow imaging based on speckle tracking is traditionally called echo-PIV (echographic particle image velocimetry) when microbubbles are used to increase the intensity of the scattered blood signal. VFI can also be achieved without contrast agent by tracking the speckles emerging naturally from blood. This approach is generally referred to as speckle tracking and may require temporal high-pass filtering to remove the high-amplitude signals arising from surrounding tissues. Ultrasound-based flow speckle tracking and echo-PIV both originate from laser speckle velocimetry and particle image velocimetry (PIV), two optical methods of flow visualization for experimental fluid dynamics. These optical techniques emerged in the early eighties [36], [37], their main difference being the number of seeded light-scattering particles. Optical PIV is now a commonly used indirect technique for measuring two or three velocity components in experimental fluid dynamics. It takes closely timed images and estimates the distance that tracer particles traveled within this time. From the known time interval and the measured displacements, the instantaneous velocity fields are calculated.

With the objective of obtaining velocity vector fields in blood vessels, laser speckle velocimetry has been adapted to ultrasound imaging as early as 1987 by Trahey et al. [7], [38]. In this study, the gain was set at its maximum to generate sufficient blood echoes for further tracking. Ultrasound vector flow imaging by speckle tracking became popular, 20 years later, with the advent of echo-PIV [39], [40]. Echo-PIV with contrast agents is now essentially utilized in clinical research to analyze the dynamics of the vortices that form in the left ventricle during diastole [41], [42], [43], [40]. Optical PIV experimenters have also shown a strong interest in echo-PIV for non-medical applications [44]. In the following, echoPIV and speckle tracking will be both called speckle tracking unless otherwise specified.

Speckles refer to interference patterns created by the presence of many sub-resolution scatterers. The texture of the speckle patterns does not correspond to underlying structure. The local brightness of the speckle pattern rather reflects the local echogenicity of the underlying scatterers, which can be used to estimate motion. Speckle tracking can be carried out on the beamformed RF (radio-frequency) signals or on the log-compressed real envelopes (B-mode images). RF signals are generally preferred over B-mode images in the special case of elastography, since small tissue displacements must be measured [45], [46], [47]. Speckle tracking is based on the hypothesis that local echogenicity is sufficiently preserved from one frame to the subsequent one. The speckle displacements are commonly determined by means of optical flow [48], [49] or block-matching techniques [50], [48], [51]. Blockmatching is usually better adapted to extract the relatively large displacements occurring in blood flows. Although several block-matching algorithms have been inspired from computer vision, the normalized cross-correlation [48], [50] is the most applied approach in ultrasound imaging. Speckle tracking is basically a three-step process: 1) subdivision of two successive images into small windows, 2) normalized cross-correlation of the window pairs, 3) peak fitting and estimation of the displacements (see Fig. 2). Normalized cross-correlations can be conveniently performed in the Fourier domain. Let $I_{a}$ and $I_{b}$ represent two successive B-mode images. These two images are both subdivided into small evenly-spaced $m \times n$ windows, $w_{a}^{k}$ and $w_{b}^{k}$, with $k=1 \ldots N$ and $N$ the total number of windows. These windows can overlap (preferably no more than 50\%) to adjust the resolution of the output velocity field. Let $W_{a}^{k}=\mathscr{F}\left(w_{a}^{k}\right)$ and $W_{b}^{k}=\mathscr{F}\left(w_{b}^{k}\right)$ be the 2-D Fourier transforms of both windows. The FFT-based normalized crosscorrelation (phase correlation) for each window $k$ is given by:

$$
N C^{k}=\mathscr{F}^{-1}\left(\frac{W_{a}^{k} \bullet \overline{W_{b}^{k}}}{\left|W_{a}^{k} \bullet \overline{W_{b}^{k}}\right|}\right)
$$

The inverse FFT is denoted by $\mathscr{F}^{-1}$. The overbar stands for the complex conjugate. In this equation, the multiplications indicated by $\bullet$ and divisions are element-wise. The relative translation (in pixels) between the two windows is given by the location of the peak in $N C^{k}$ :

$$
\left(\Delta i_{a \rightarrow b}^{k}, \Delta j_{a \rightarrow b}^{k}\right)=\arg \max _{i, j}\left(N C^{k}\right)
$$

The position of the correlation peak can be determined with sub-pixel accuracy. A robust technique is to fit the correlation peak to some function, such as a parabolic or Gaussian curve [52], [53]. The velocity vectors are finally deduced using the pixel size and the ultrasound pulse repetition period. Vector flow imaging by speckle tracking has been successfully applied in a number of human cardiovascular flows, mostly in the carotid [44], [54] and the left ventricle [42], [43], [55]. The above mentioned algorithm is given in its simplest form. Standard state-of-the-art numerical methods developed for optical PIV can be applied to increase the precision. To cite a few, these techniques include multiple-pass and ensemble crosscorrelation, coarse-to-fine analysis, and interrogation window deformations [56]. Since a large number of frames can be available, high-frame-rate vector flow imaging can greatly benefit from these algorithms. Finally, robust regularization is an essential final step if differential quantities must be derived (e.g. vorticity, shear rates and pressure gradients). Numerous basic to advanced algorithms exist in the literature to regularize velocity fields [56], [57], [58], [40].

The main clinical limitation for echo-PIV (speckle tracking with contrast-enhanced echography) is the intravenous administration of gas-filled microbubbles. In clinical practice, contrast-enhanced ultrasonography can be required when an echocardiographic examination is difficult, such as in patients with obesity or lung disease. Although no major 


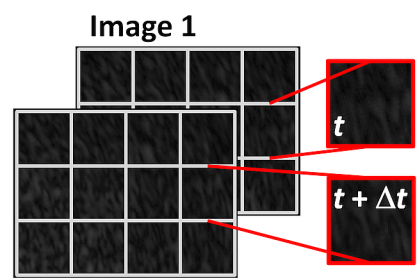

Image 2

\section{Subdivide images into windows}

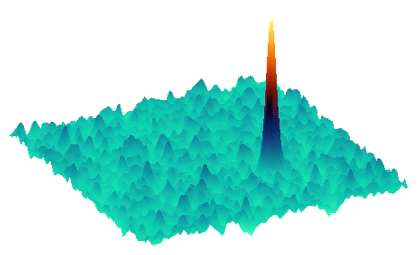

2. Cross-correlation + Peak fitting

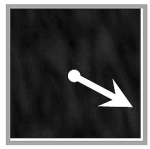

\section{Displacement vectors}

Fig. 2. The three basic steps of cross-correlation-based speckle tracking for vector flow imaging. Advanced tracking also includes multiple-pass and ensemble cross-correlation, coarse-to-fine analysis, and interrogation window deformations

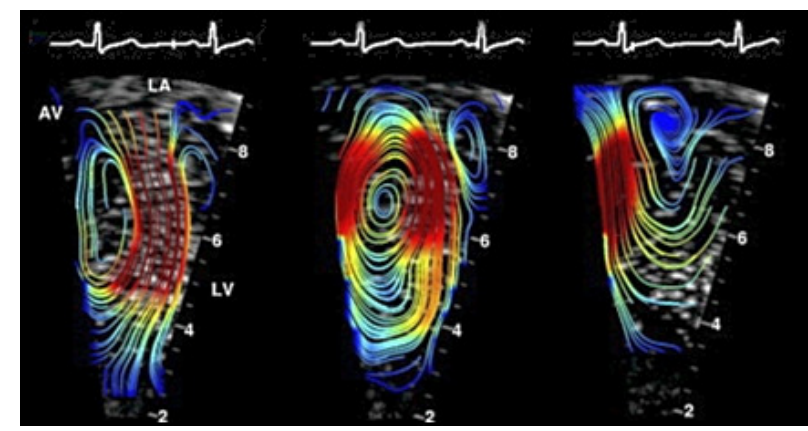

Fig. 3. Left intraventricular flow by echo-PIV with contrast agents. Adapted from [60]. By way of comparison, see Fig. 8 in paper II, obtained without contrast agent by Fadnes et al. [61].

side-bioeffect has been noticed in well-controlled situations, contrast-enhanced ultrasonography is relatively time- and staffconsuming and thus cannot be routinely recommended. Before becoming an accepted clinical examination, multi-center trials are required to demonstrate the efficiency of echo-PIV in terms of cost-effectiveness and clinical outcomes.

Echo-PIV with contrast agents is now essentially utilized in clinical research to analyze the dynamics of the vortices that form in the left ventricle during diastole [41], [42], [43], [59], [40] (see Fig. 3).

\section{TRANSVERSE OSCILLATION}

The underlying mechanism for finding the axial velocity component using (1) is the ultrasound oscillation with a frequency of $f_{0}$. This makes it possible to detect the frequency or phase shift. Traversing the ultrasound beam in the lateral direction does not yield an oscillation, and no velocity component can, thus, be found. The basic idea introduced by Jensen, Munk and Anderson [62], [63], [8], [64], [9] is to make a transverse oscillation (TO) in the ultrasound field as shown in Fig. 4. A motion transverse to the ultrasound beam then yields an oscillation with a frequency proportional to the transverse velocity.

The transverse oscillation can be generated by having a receive apodization with two peaks as shown in the top of Fig. 4. There is a Fourier relation between the transducer's apodization function and the ultrasound field at the focus or in the far-field [65]. Having two peaks, thus, generates a sinusoidal oscillation, and the shape of the peaks will influence the width of the resulting field. The lateral oscillation
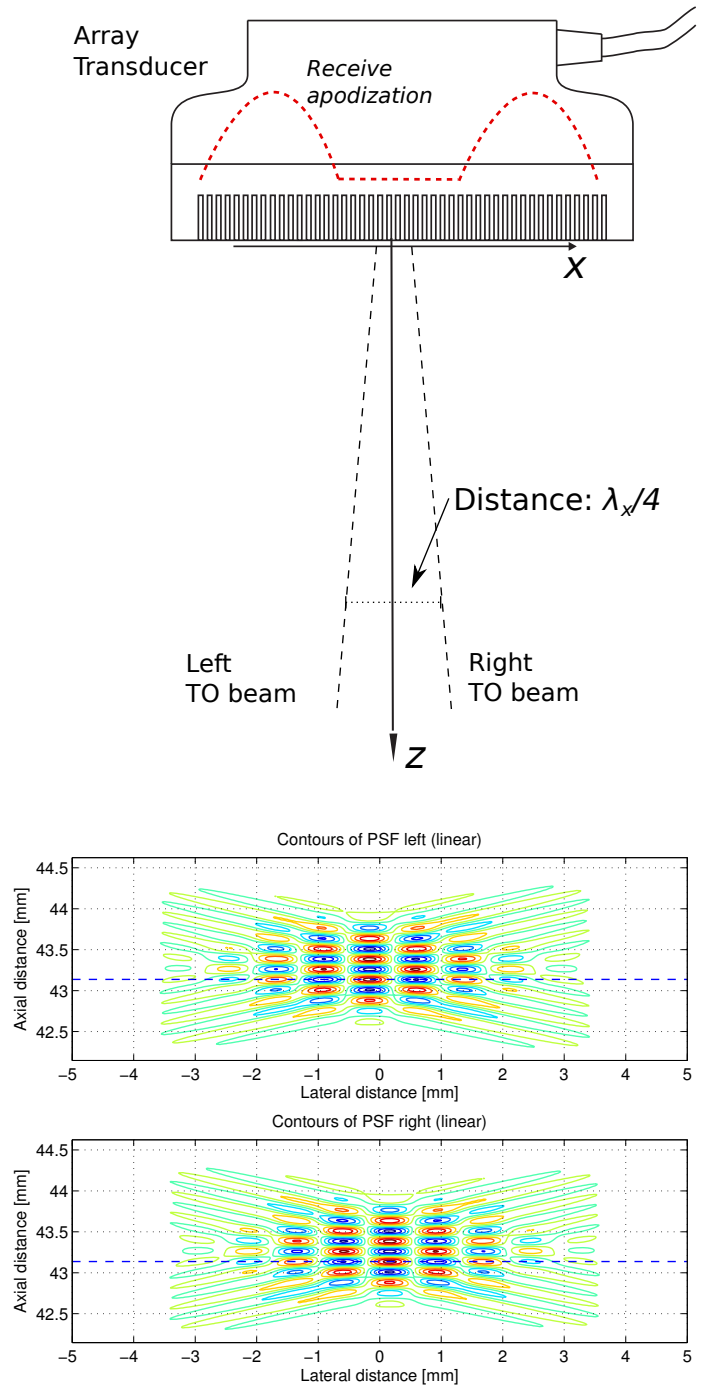

Fig. 4. Generation of transverse oscillating field. The top graph shows the apodization applied on the linear array transducer for generating to two beams. The bottom graphs show the point spread functions of the left and right TO field.

wavelength $\lambda_{x}$ is given by [8]:

$$
\lambda_{x}=\frac{2 \lambda d}{P_{d}}
$$

where $d$ is depth in tissue and $P_{d}$ is the distance between the peaks.

In a traditional velocity estimation system a Hilbert transform is conducted on the received signal to yield a one-side 
spectrum from which the sign of the velocity can be deduced [4]. In TO, a spatial quadrature signal can be generated by focusing two parallel beams displaced by a quarter wavelength $\lambda_{x} / 4$ to have a 90 degree phase shift between the beams [8]. An alternative is to Hilbert transform the apodization waveform as was performed by Anderson [9]. The resulting beams should form a Hilbert transform pair and any deviations from this results in a bias on the estimates. Several schemes for optimizing the point spread function (PSF) have therefore been suggested using dynamic apodization [66], [67], spatial quadrature [68], synthetic aperture imaging [69], plane wave imaging [70], and optimization of the TO field [71]. A new approach is to focus directional beams perpendicular to the ultrasound propagation direction and then Hilbert transform this signal [72], [73]. The purpose of all these approaches is to optimize the TO PSF and thereby reduce the bias and standard deviation.

The axial and lateral velocity components are estimated using the four samples acquired in each emission emanating from the complex signals in the two parallel beams. This can be performed using the conventional autocorrelation estimation [74], [8] or a heterodyning demodulation technique as suggested by Anderson [75], where the received signals are multiplied to reduce the influence of the axial velocity component on the lateral component estimation. A special fourth order estimator was developed by Jensen [76], which yields the two components independently as ${ }^{1}$ :

$v_{x}=\frac{\lambda_{x}}{2 \pi 2 T_{p r f}} \arctan 2\left(\frac{\mathfrak{I}\left\{R_{1}(1)\right\} \mathfrak{R}\left\{R_{2}(1)\right\}+\mathfrak{I}\left\{R_{2}(1)\right\} \mathfrak{R}\left\{R_{1}(1)\right\}}{\mathfrak{R}\left\{R_{1}(1)\right\} \mathfrak{R}\left\{R_{2}(1)\right\}-\mathfrak{I}\left\{R_{1}(1)\right\} \mathfrak{I}\left\{R_{2}(1)\right\}}\right)$

and

$v_{z}=\frac{\lambda}{2 \pi 4 T_{\text {prf }}} \arctan 2\left(\frac{\mathfrak{I}\left\{R_{1}(1)\right\} \Re\left\{R_{2}(1)\right\}-\mathfrak{I}\left\{R_{2}(1)\right\} \mathfrak{R}\left\{R_{1}(1)\right\}}{\mathfrak{R}\left\{R_{1}(1)\right\} \mathfrak{R}\left\{R_{2}(1)\right\}+\mathfrak{I}\left\{R_{1}(1)\right\} \mathfrak{I}\left\{R_{2}(1)\right\}}\right)$.

Here $R_{x}(1)$ is the lag one complex autocorrelation of signals combined from the four measurements [76], where $\mathfrak{I}$ denotes imaginary part and $\Re$ real part. $T_{p r f}$ is the time between pulse emissions. This estimator has been found to be less sensitive to noise than the heterodyning demodulation method [77]. A phase-based block matching method for finding the displacement for TO fields was developed by Basarab et al [78]. Another approach based on the instantaneous signal phase and multidimensional autocorrelation was developed by Sumi [79], so several different approaches to find the velocity components exist. Anderson [80] also showed the similarity between TO and dual beam velocity estimation for a monochromatic field using a number of approximations.

An inital in-vivo measurement with the TO method is shown in Fig. 5 of the carotid artery bifurcation. The image is acquired right after peak systole and shows a vortex forming at the lower branch in the bifurcation. This is a common finding in healthy human volunteers. and the vortex appears in the image for around $100 \mathrm{~ms}$ before a laminar flow again is attained in the lower branch during the diastolic phase. The

\footnotetext{
${ }^{1}$ The $\arctan 2$ functions should be used to find the phase angle, where the numerator is input as one argument and the denominator as a second argument. Hereby the angle can be found in the range $\pm \pi$, thus, preserving the full velocity range.
}

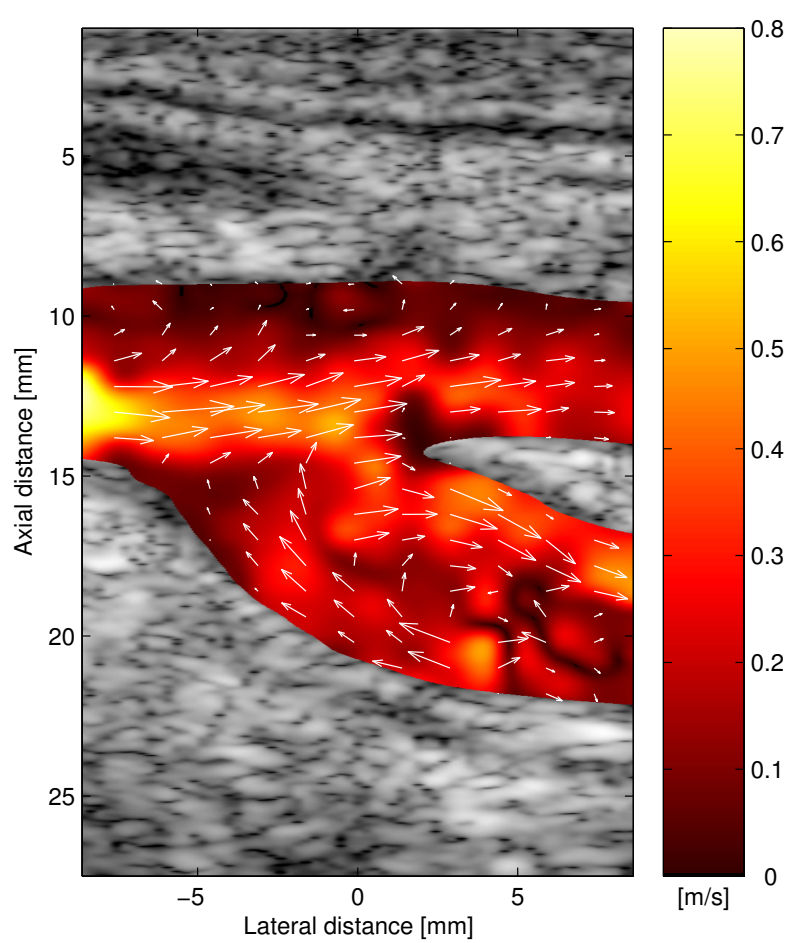

Fig. 5. TO vector flow image from the carotid artery bifurcation. The arrows show the velocity direction and magnitude, and the magnitude is also show by the color intensity (from [81]).

image demonstrates several benefits of vector flow imaging. The flow in the upper branch has a beam-to-flow angle close to $90^{\circ}$, where velocities are almost impossible to accurately measure with a traditional system. The angles vary as a function of space and time and angles from 0 to $360^{\circ}$ can be found, thus, making angle correction impossible, as it has to be conducted independently for all positions and all time. Further, the arrows clearly show flow direction and magnitude, and the color also shows the velocity magnitude in the correct range for the carotid artery.

The TO method was initially developed for linear array probes [8], [9], but has also been further developed for phased [82] and convex array probes [71]. A 3-D implementation has also been made [83], [84] and is discussed further in Section VIII.

\section{DIRECTIONAL BEAMFORMING}

Time shift estimation is one of the two "main-stream" flow estimation techniques for color flow imaging together with phase-shift estimation [4]. Time-shift estimation using crosscorrelation has been used since 1970s to estimate the axial component of the blood flow [85], [86], [87], [88], [89], [90].

Consider a single scatterer positioned in front of the transducer at depth $z=z_{0}$. A pulse is sent at time $t_{0}$ and the received signal $w_{0}(t)$ is recorded. Then at a time $t_{1}=t_{0}+T_{p r f}$ a second pulse is emitted and the echo $w_{1}(t)$ is recorded. $T_{p r f}$ is the time between pulse emissions. In both cases, the time $t$ is relative to the origin of the transmission. If the scatterer is moving, then the second echo will be shifted by $t_{s}$ from the first echo 
given by:

$$
t_{s}=\frac{2 v_{z}}{c} T_{p r f}
$$

The correlation function of the two RF lines is:

$$
R_{12}(\tau)=\int_{T} w_{1}(t) w_{2}(t+\tau) d t
$$

If the scatterer does not move, then $w_{1}(t) \equiv w_{2}(t)$, and $R_{12}(\tau)$ is the auto-correlation of $w_{1}(t)$ with a maximum at lag $\tau=0$. When the scatterer moves axially at a distance $\Delta z=v_{z} T_{p r f}$ between the two emissions, then the second signal $w_{2}(t)$ represents a time-shifted version of $w_{1}(t)$ [4], [90]:

$$
w_{2}(t)=w_{1}\left(t-t_{s}\right) .
$$

The correlation function between $w_{2}(t)$ and $w_{1}(t)$ is equal to a time shifted version of the auto-correlation function of $w_{1}(t)$ :

$$
\begin{aligned}
R_{12}(\tau) & =\int_{T} w_{1}(t) w_{2}(t+\tau) \\
& =\int_{T} w_{1}(t) w_{1}\left(t-t_{s}+\tau\right)=R_{11}\left(\tau-t_{S}\right) .
\end{aligned}
$$

The autocorrelation $R_{11}\left(\tau-t_{s}\right)$ has a maximum when the argument is equal zero, i.e. when $\tau=t_{s}$. The axial velocity estimate $\hat{v}_{z}$ is then:

$$
v_{z}=\frac{c}{2} \frac{\hat{t}_{s}}{T_{p r f}}
$$

where $\tau=\hat{t}_{s}$ is the lag at which the estimated cross-correlation function $\hat{R}_{12}(\tau)$ has a maximum.

A weakly focused transmit beam can span several image lines, and an unfocused beam, as in the case of plane-wave or spherical wave transmits, can span the whole region of interest. In this case it is possible to track the shift of the position of the correlation function not only along a single line, but across lines as suggested by Bonnefous [91].

Fig. 6 illustrates the case where a single moving scatterer is scanned. The top subplot shows two frames. The scatterer moves at a distance $\lambda / 8$ along the beam (axially) and $3 / 4 \lambda$ laterally between the two frames. The motion is sought for the center line (index 0). Subplot 6(b) shows the correlation of the line with index 0 from the first frame with the same line (index 0 ) from the second frame as well as with the neighboring lines $(-1,+1)$. Notice that the cross-correlation in this case is normalized only to the energy in the center line.

It can be seen that the position of the axial peak for all three correlation functions is at the same lag. The algorithm described by Bonnefous in [91] is as follows:

1) estimate the cross-correlation of the center line only for multiple lags $\left(R_{0,0}(u)\right)$

2) find the lag of the peak of the cross correlation $u_{s}$

3) calculate the correlation coefficients at lag $u_{s}$ with neighboring lines $R_{0,-1}\left(u=u_{s}\right)$ and $R_{0,+1}\left(u=u_{s}\right)$

4) fit a parabola to $R_{0,0}\left(u_{s}-1\right), R_{0,0}\left(u_{s}\right)$, and $R_{0,0}\left(u_{s}+1\right)$ to interpolate the sub-sample axial displacement [89]

5) fit a parabola to $R_{0,-1}\left(u_{s}\right), R_{0,0}\left(u_{s}\right)$, and $R_{0,+1}\left(u_{s}\right)$ to find the lateral motion.

For this method to work, the transmit field must be uniform across the beams, and the author suggests the use of plane
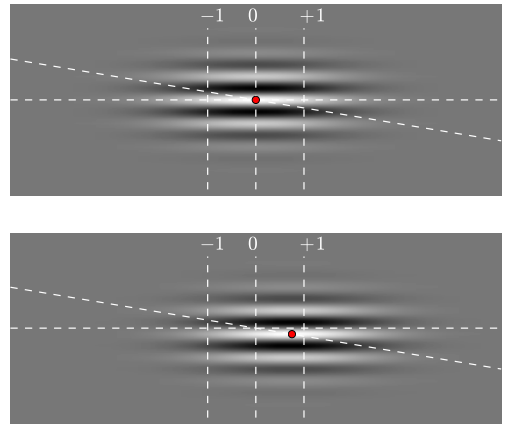

(a)

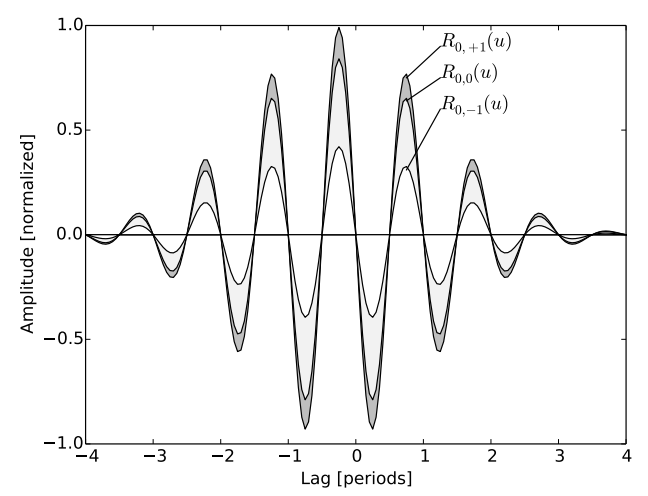

(b)

Fig. 6. A single scatterer moving axially at a distance $\lambda / 8$ and laterally at a distance $3 / 4 \lambda$. Subplot (a) illustrates the change in the 2D image of the scatterer and subplot (b) shows the cross-correlation function of the center line (0) from the first frame with the neighbouring lines from the second frame.

waves. The beams in lateral direction, which are used for tracking are one wavelength apart.

In the analysis of the precision of the time-shift estimation, Foster et al. show that the largest source of error is the flow gradient in the averaging region for the cross-correlation function [90]. Jensen suggested selecting a correlation kernel along the flow [92], [93], [94], which makes it possible to estimate the vector flow, and minimizes the errors introduced by the flow gradient.

One way to sample the acoustic field along the flow direction is to use beamforming. The region of interest for the flow estimation must be within the focal zone of the transmit beam, in which case the wavefront is close to planar $^{2}$.

The focusing points are given by [94]:

$$
\vec{r}_{p}(k)=\left[k \Delta x^{\prime} \sin (\theta)+x_{s t}, 0, k \Delta x^{\prime} \cos (\theta)+z_{s t}\right],
$$

where $\Delta x^{\prime}$ is the spatial sampling interval, $k$ is the sample index, $\theta$ is the angle between the flow vector and the $z$ axis, and $\vec{r}_{s t}=\left(x_{s t}, 0, z_{s t}\right)$ is the point in the image for velocity estimation. This signal passes through the center of a coordinate system whose origin is at $\vec{r}_{s t}$, and the sample index $k$ can be both negative and positive in the interval $\left[-N_{k} / 2, N_{k} / 2\right]$. The number of beamformed samples in this case is $N_{k}+1$.

\footnotetext{
${ }^{2}$ Strictly speaking, this is not true, but the phase errors in the focal region are below $\lambda / 8$ and the diffraction is constructive.
} 
The propagation time from the origin $\vec{r}_{o}(i)$ of transmission $i$ to the point $\vec{r}_{p}(k)$ and back to position $\vec{r}_{e}(j)$ of receiving element $j$ must be found to focus a signal at spatial location $\vec{r}_{p}(k)$. The propagation time in receive is determined by the geometric distance between the sample location and the receive element:

$$
t_{r}(k, j)=\frac{\left|\vec{r}_{e}(j)-\vec{r}_{p}(k)\right|}{c} .
$$

The estimation location $r_{s t}$ is on the axis of the transmit beam, hence the propagation time from the origin of emission to point $\vec{r}_{p}(k)$ is:

$$
t_{e}(k)=\frac{1}{c}\left(\left|\vec{r}_{s t}-\vec{r}_{o}(i)\right|+k \Delta x \cos (\theta)\right) .
$$

The directional signal at acquisition $i$ is formed as:

$$
g_{i}(k)=\sum_{j=0}^{N_{r}} g_{r}\left(j, t_{e}(k)+t_{r}(k, j)\right),
$$

where $g_{r}(j, t)$ is the signal received by element $j$ at time instance $t$ from the start of the emission.

The processing for the directionally beamformed signals follows the steps for the time-shift estimation. The cross correlation between the signals $g_{i}$ and $g_{i+1}$ acquired at $T_{p r f}$ seconds apart is:

$$
\begin{aligned}
R_{12}(l) & =\frac{1}{N_{k}+1} \sum_{k=-N_{k} / 2}^{N_{k} / 2} g_{i}(k) g_{i+1}(k+l) \\
& =\frac{1}{N_{k}+1} \sum_{k=-N_{k} / 2}^{N_{k} / 2} g_{i}(k) g_{i}\left(k+l-k_{s}\right)=R_{11}\left(l-k_{S}\right),
\end{aligned}
$$

where the scatterer has moved $k_{s}$ samples between the two acquisitions and $R_{11}(l)$ is the autocorrelation function of the directional signal. A global maximum is found at $l=k_{s}$, and the velocity is:

$$
|\vec{v}|=k_{s} \frac{\Delta x}{T_{p r f}} .
$$

The directional beamforming approach has been shown to improve the velocity estimates compared to TO VFI [95] due to the higher correlation along the flow direction. The angle for the beamforming has to be known, and this can be found from the normalized correlation function [96]. Beams are made for a number of directions and the normalized correlation functions are found. The highest normalized correlation is found, when the flow is tracked in the correct direction, and this indicates the correct beam-to-flow angle. Beams are then focused in this direction, and the velocity magnitude is found. The approach yields very accurate results, but is quite computationally intensive.

\section{SPECTRAL BASED Methods}

Pulsed-wave (PW) and continuous-wave (CW) system estimate the frequency shift and display it as a function of time in the form of a spectrogram [4]. The echoes created by a scatterer moving with speed $v$ probed with a monochromatic plane wave exhibit a frequency shift $f_{D}$ given by (1). Experiments with constant flow (for example using a thread [97]) demonstrate spectral broadening. This effect has been studied

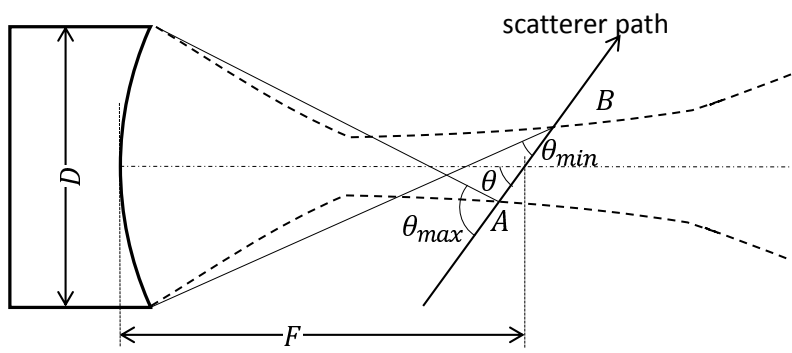

(a)

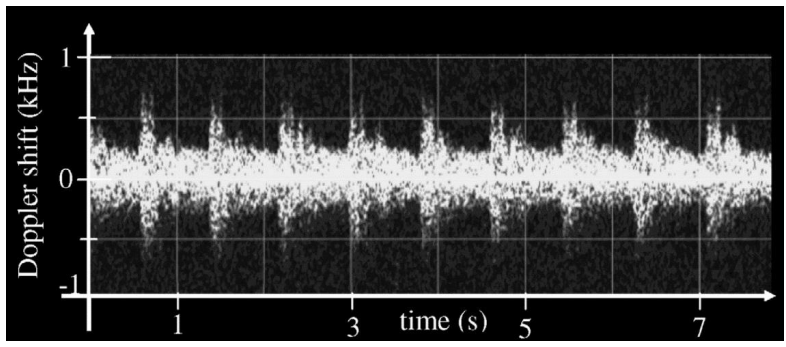

(b)

Fig. 7. Dependence of ultrasound Doppler bandwidth on beam geometry: (a) beam geometry used to derive the relation; (b) typical spectrogram obtained from a transversely insonated common carotid artery (from [16])

theoretically and experimentally in the 1980s by Newhouse and colleagues [98], [99], [100], [101]. These studies show that for a plug flow, the main cause for the spectral broadening is caused by the angle between the flow direction and the rays to the different parts of the transducer, which varies in the range $\left[\theta_{\min }, \theta_{\max }\right]$ (see Fig. 7), where $\theta_{\min }$ and $\theta_{\max }$ are the angles between the flow direction and the outer edges of the ultrasound field.

The model developed in these studies states that the spectrum is symmetric, and that the bandwidth $B_{D}$ is

$$
B_{D} \simeq \frac{2}{\lambda} \cdot \frac{D}{F} \cdot v \cdot \sin (\theta),
$$

where $D$ is the diameter of a circular focused transducer, and $F$ is the focal distance.

Using (1) and (21) it can be seen that if a flow is scanned at an angle $\theta=90^{\circ}$, then the frequency shift $f_{D}$ is 0 , and that the bandwidth will be proportional to the velocity magnitude v. Fig. 7(b) shows a spectrogram obtained from a transversely insonated common carotid artery [16]. The spectra are centered at $0 \mathrm{~Hz}$ and the bandwidth follows the change in velocity $v$.

In the early 1990s Newhouse and colleagues suggested the design of vector-flow systems that use the frequency shift as an estimate of the axial velocity component and the spectral bandwidth as the lateral component of the flow (across the beam) [102]. The feasibility of the approach was experimentally verified by Tortoli and colleagues [103], [104].

One of the weaknesses is that other factors contribute to spectral broadening [105], for example flow gradient in the vessel and acceleration. While spectral bandwidth correlates with velocity (see Fig. 7(b)), it does not yield accurate results.

Tortoli and colleagues suggested a system that utilizes the symmetry properties of the spectrum to get two independent 


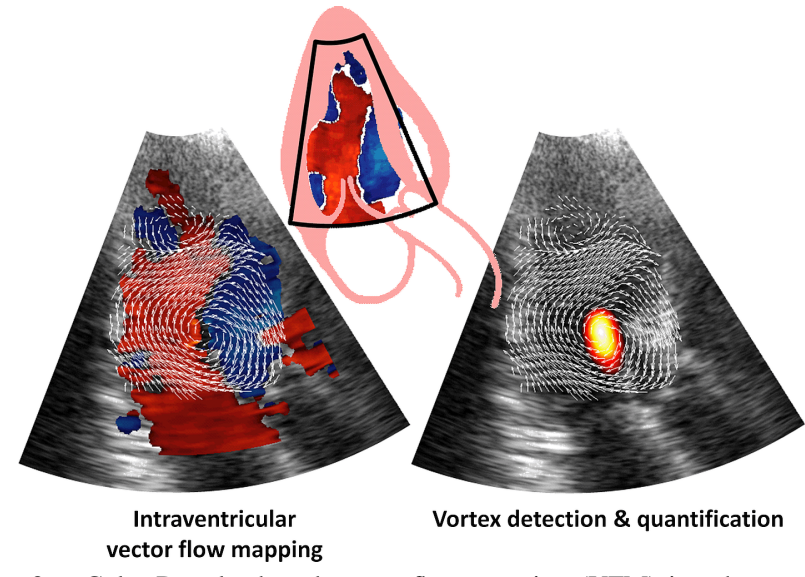

Fig. 8. Color-Doppler based vector flow mapping (VFM) in a human left ventricle using the technique described in [109] (with a conventional GE scanner). VFM allows vortex detection and quantification (right panel) [110].

measures - one for the beam-to-flow angle $\theta$ and the other for the axial component $v_{z}$ [16], [106]. The approach uses two beams, each beam giving one spectrum, transverse and axial, respectively. The beam for the transverse spectrum is steered till the spectrum becomes symmetric (as shown in Fig. 7(b)). When the spectrum is symmetric, the beam is perpendicular to the flow, thus giving an estimate for the flow angle. The axial beam is also steered to get a favorable beam-to-flow angle that yields high accuracy. Commercial implementations of this approach exist.

The spectral bandwidth approach has also been suggested for finding the out-of-plane velocity component [107]. The technique requires calibration to work, and currently the lateral component has to be negligible for the method to be effective.

The transverse velocity spectrum can also be found using a TO ultrasound field as described in [108]. A fourth order correlation approach is then used to calculate the velocity spectrum in the transverse direction, and conventional spectrum in the axial direction yields the velocity distribution for the axial component. The approach works for beam-to-flow angles above $60^{\circ}$, where conventional spectral estimation is highly unreliable. Above this angle the approach can be used to compensate the axial spectrum to give quantitative velocity values.

\section{COLOR-DOPPLER BASED VECTOR FLOW MAPPING}

Color-Doppler-based vector flow imaging (named as vector flow mapping, VFM, in the literature) is another technique for vector flow imaging, in the left ventricular cavity specifically. This flow imaging method is based on the post-processing of color Doppler images (see Fig. 8). It was first proposed by Ohtsuki and Tanaka in 2006 under the appellation of echodynamography [111], [112]. Another numerical model, now implemented in Hitachi ultrasound scanners [113], was then developed by Garcia et al. in 2010 [109]. VFM has been compared with optical PIV in a realistic left ventricle model, and with MRI in the left ventricular 3-chamber view of a few subjects [109]. It minimizes the 2-D velocity divergence in the plane of interest. In a polar coordinate system, the 2-D velocity divergence writes as $r \partial_{r} V_{r}+V_{r}+\partial_{\theta} V_{\theta}=0$, where $\left(V_{r}, V_{\theta}\right)$ are the velocity components with coordinates $(r, \theta)$. In the polar system associated to the phased array, and assuming that the flow is two-dimensional, the cross-beam (angular) velocity components $V_{\theta}$ can be thus deduced from:

$$
\partial_{\theta} V_{\theta}=r \partial_{r} V_{D}+V_{D}
$$

where $V_{D}\left(=-V_{r}\right)$ represents the Doppler velocities. In practice, the 2-D flow assumption partly fails, so that equation (22) is ill-posed. By constraining the boundary conditions and regularizing the system in (22), it becomes overdetermined and can be solved in the least-squares sense using a finite difference method. In [109], the wall velocities were assumed tangential to the endocardium. Under these conditions, a simplified regularized form of (22) can be given by:

$$
v_{\theta}=\arg \max _{V_{\theta}}\left\{\left\|\partial_{\theta} V_{\theta}-r \partial_{r} V_{D}-V_{D}\right\|^{2}+\lambda\|\vec{V} \cdot \vec{n}\|_{\Gamma}^{2}\right\}
$$

In (23), $\vec{n}$ represents a unit vector normal to the endocardial surface $\Gamma$, and $\lambda$ is the regularization parameter. Note that a penalty term (low-pass operator) may be added in the cost function in (23) to enforce smoothness of the underlying vector field. In comparison with the other vector flow imaging techniques described in this paper, VFM does not provide a true planar projection of the 3 -D velocity field. To produce a 2-D field, this technique assumes that the out-of-plane components are small. VFM is readily available in Hitachi scanners and has been tested in several clinical situations [114], [115], [116]. It has also been assessed in patients with echocardiographic data extracted from GE (General Electric Healthcare) Vivid scanners [117], [118], [119], [120]. It appears from these clinical studies that the analysis of the diastolic vortex dynamics could improve diastology. Since wall boundary conditions are necessary, VFM requires a relatively large scan sector to enclose the whole left ventricle. This may reduce the frame rate to $<10$ frames/s with conventional sequential color Doppler. At least five heart cycles must, thus, be registered to obtain the full dynamics of the intracardiac flow, which can make VFM challenging in patients with cardiac arrhythmias and/or breath-holding difficulty. VFM has not yet been evaluated with high-frame-rate color Doppler. As already mentioned, clutter filtering will be a concern to be solved with unfocused waves. Another issue is the presence of aliasing in color Doppler. Range cross-correlation techniques [121], [96] or staggered transmit sequences [122] can provide solutions to this problem.

\section{3-D VECTOR FLOW IMAGING}

The methods mentioned above all estimate the 2-D velocity vector, but the flow is inherently $3-\mathrm{D}$ in nature. Often it is assumed that the out-of-plane motion is negligible, but for complex flow found in tortuous vessels, in stenoses, and bifurcations there can be significant out-of-plane velocities. It is therefore natural to extend the abovementioned techniques to estimate the third components by enabling beamforming in the elevation direction.

The crossing-beams method was extended by Fox and Gardiner to yield the full velocity field [123]. Three probes 
where used and the frequency shift detected for all probes, which could then be combined to find the velocity magnitude and angles. Gomez et al [124] combined three or more conventional color flow maps to determine the 3-D vector velocity using a least-squares approach. The method was used on three young patients under sedation and could display 3-D velocity fields through e.g. the aortic valve and flow from the left atrium into the left ventricle.

Bonnefous extended the cross-correlation approach to three directions by focusing beams in the axial, lateral and elevation direction [91] to perform correlations in the three directions to find all velocity components. The approach was only investigated for flow in one direction.

Another technique for making 3-D velocity estimation was developed by Newhouse et al. [102]. They used two receiving transducers and deduced the full velocity magnitude of a string phantom by estimating both the spectral shift and the bandwidth of the spectrum for both transducer signals.

Hein used the correlation between parallel beams to find the transit time and hence velocity between three parallel beams [125]. A combined transducer with three crystals was used for emitting and receiving the three beams, and it was shown that measured peak flow velocities were accurate within $15 \%$ and angles within $\pm 5^{\circ}$.

Speckle tracking has also been translated to 3-D [126]. Currently it has mostly be applied on tissue motion tracking of e.g. the myocardium [127], [128].

The introduction of 2-D matrix probes has made it possible to focus beams in all directions, and this was used by Pihl and Jensen [129], [83] to developed a 3-D version of TO. A $32 \times 32$ elements probe emitted a focused field and 5 beams were focused simultaneously in receive from each emission. A transverse oscillation was generated in receive by a dual peak apodization along either the $x$ or $y$ directions and gave fields oscillating in these directions. A modified estimator could then yield the full 3-D velocity vector with a low bias and a precision around $8 \%$ to $16 \%$ for parabolic flow in a flow rig [84]. In-vivo data has also been acquired using this approach [130], and an example is shown in Fig. 9 from the carotid artery. The 3-D velocity vector has been found in two orthogonal planes. The predominate component is in the $x$ direction through the imaging plane, which cannot be estimated using a 2-D VFI systems (see bottom figures in Fig. 9).

\section{Flow Vector Visualization Algorithms}

Display of vector flow images is challenging as both velocity magnitude and direction have to be shown. They both change rapidly throughout spatial position and over time. Several methods for the visualization have therefore been developed and are described below.

\section{A. Arrow Displays}

After deriving the flow vector maps, the next step is to display them onto the screen. One common approach that has been used for a number of years is to render each flow vector as an arrow within the image frame [131], [132]. For this
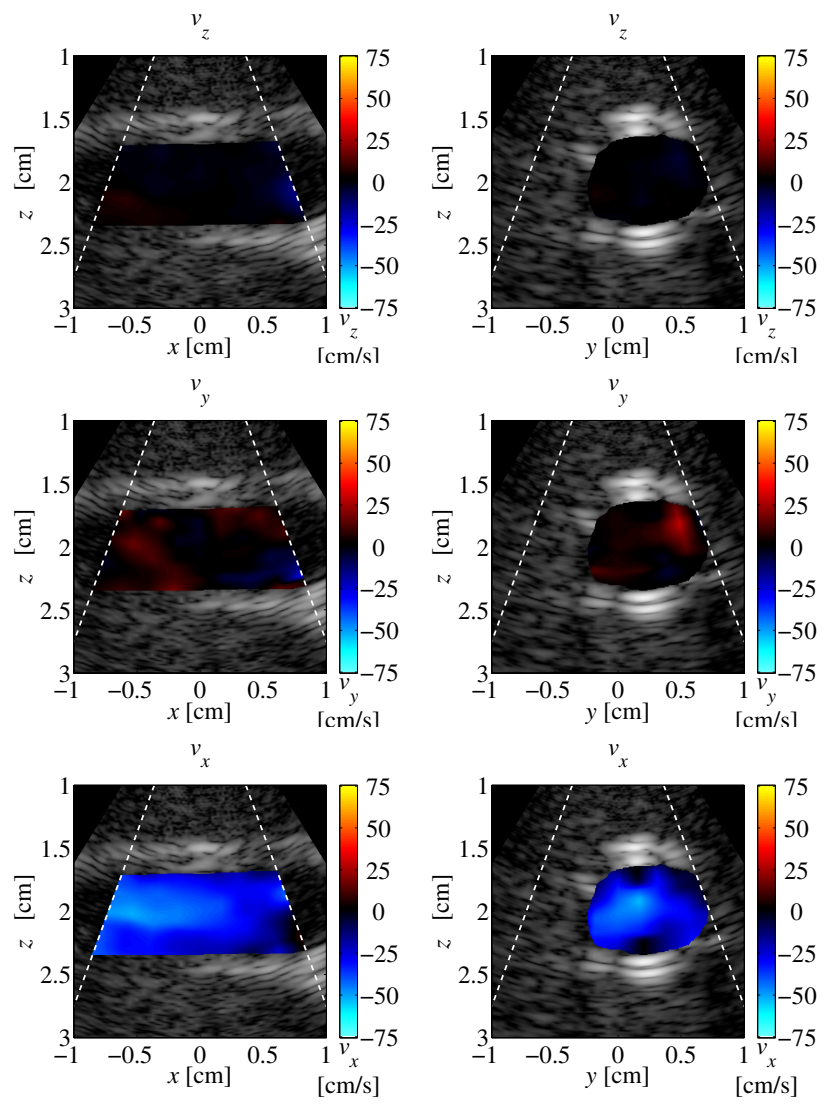

Fig. 9. In-vivo scan of the carotid artery of a healthy volunteer. Two orthogonal planes are acquired (left and right images) and the velocity is estimated for all three directions $\left(v_{z}, v_{y}, x_{x}\right)$. A 2-D vector flow system would not detect the $v_{x}$ component (from [130]).

vector visualization approach, an image grid is first created as similar to conventional color flow imaging, and the power detected after echo canceling [4] at each pixel position is used to form a flow display mask. If a pixel falls within the flow display mask, its corresponding flow vector will be displayed as an arrow at that position. The length of the flow arrow is generally set forth according to the flow velocity magnitude (i.e. the norm of axial and lateral velocity estimates), and the arrow's pointing direction is set based on the flow angle (mapped from the axial and lateral velocity values). Often times, this arrow display is overlaid on top of the color flow image [133] or a color-coded velocity magnitude map [81].

Published images typically include straight tube phantoms with steady flow profiles [134] and pilot in-vivo images of the human carotid artery [81], [45], [29], [133], [135]. This display approach has also been used in both single-line, multigate Doppler methods [136] and those that render image maps of flow vectors [133]. The shown images can generally depict the parabolic flow profile inside straight tubes as expected from fluid mechanics principles, while the in-vivo data have concurred the multi-directional nature of flow at the carotid bifurcation. Note that the flow arrows themselves can be color coded too, as demonstrated in a cardiac imaging study [109]. 


\section{B. Color Map Displays}

Another display approach is to map flow vectors to a multicolor palette that encodes both velocity magnitude and flow angle information. Instead of using the typical hot-cold color map used to encode velocity values in color flow imaging, flow vector color mapping would respectively assign, on a pixel by pixel basis, the color hue based on the flow angle and the color intensity (as well as saturation) based on velocity amplitude [137], [138]. As a similar variant, the flow angle and flow velocity amplitude can be shown as two separate color maps [139], [140], [141].

It should be pointed out that the concept of displaying flow vectors as color maps may be readily integrated with the concept of flow arrow displays to yield a more integrated form of flow vector visualization [142], [143]. This visualization approach has offered an alternative way of highlighting flow vector information as compared to plain display of flow arrows or color maps. It has been commercially implemented on the BK ProFocus UltraView platform [144]. Pilot images in-vivo have been shown in the carotid arteries [145] and the ascending aorta [146], and results have shown promise.

\section{Dynamic Visualization}

Arrow displays and color mapping of flow vector estimates can be considered as more classical visualization approaches for ultrasound VFI. The common attribute of these visualization schemes is that the flow vectors are shown on a static basis in which they are always pivoted at the same pixel position. To more lucidly highlight the dynamic nature of blood flow, especially in cases with complex flow patterns, efforts have been made to dynamically update the position of flow vectors in between frames by applying particle visualization strategies that are well established in computer vision [147], [148], [149].

In one particular dynamic visualization algorithm (dubbed as vector projectile imaging) [33], flow projectiles are shown at a randomized selection of launch points within the blood vessel, and their lengths and color are both pegged to the velocity magnitude. In between frames, the position of each flow projectile would be updated by adding the corresponding inter-frame vector displacement value. The process is repeated over time, and it is coupled with randomized projectile life time and relaunching. Also, flow speckle motion is rendered as an adjunct visualization to enhance the depiction of flow trajectories (based on another method reported earlier [150]). As shown in a series of carotid bifurcation imaging experiments (see Fig. 10 for representative examples), such an approach has highlighted the spatiotemporal evolution of stenotic flow jets and recirculation zones in a visually intuitive way. It has been commercially implemented as a diagnostic mode in the new Mindray Resona 7 platform [151].

Other emerging ways of dynamic flow visualization are under development. Three efforts are particularly worth noting. One of them is the application of smoothed particle hydrodynamics principles to render the multi-directional nature of hemodynamics inside blood vessels [152]. Another is the dynamic depiction of flow trajectories as tailored pathlets to track
A
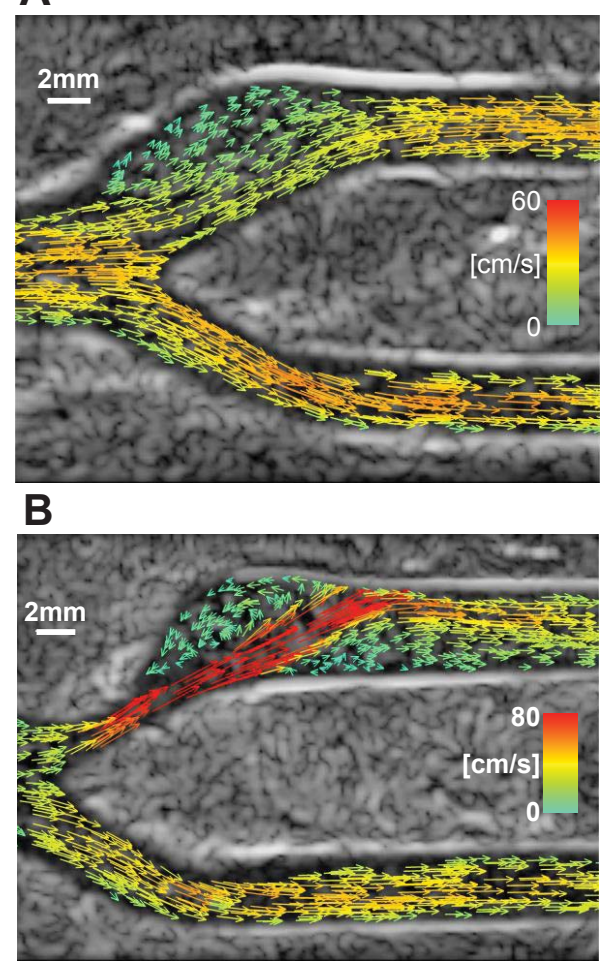

Fig. 10. Vector projectile image frames of carotid bifurcation phantom experiments. The image frame taken during peak systole is shown for (A) healthy bifurcation and (B) diseased bifurcation with $50 \%$ eccentric stenosis. Complex flow features including flow jet and recirculation in the diseased carotid bifurcation are effectively highlighted. (From [33]).

the spatio-temporal progression of systolic and diastolic flow dynamics inside heart chambers and the aorta [153], [154], [155], [117]. The latter is the computation of Lagrangian coherent structures to depict the topology of blood transport. It is conceivable that, with further maturity in the adoption of graphics processing unit technology in ultrasound imaging [34], dynamic visualization of flow vectors can gain further prominence in the near future.

\section{Clinical applications}

The number of clinical studies of vector flow imaging is still limited as only a few commercial implementations exist. The TO VFI method approach has been implemented on BK Ultrasound scanners and yields images as shown in Fig. 11 of the descending aorta. The color scale depicts direction and more quantitative information can be gained from the superimposed arrows.

\section{A. Validation of VFI imaging}

The first examples of TO VFI were reported by Udesen [81]. The method was validated against MR data for eleven healthy volunteers on the carotid artery. MR examinations were performed with a $1.5 \mathrm{~T}$ whole body scanner using a prospective ECG-triggered phase contrast sequence employing a cervical coil. The stroke volume of this was compared to the TO estimates measured on the right common carotid artery at a beam-to-flow angle close to $90^{\circ}$. The study showed a high 


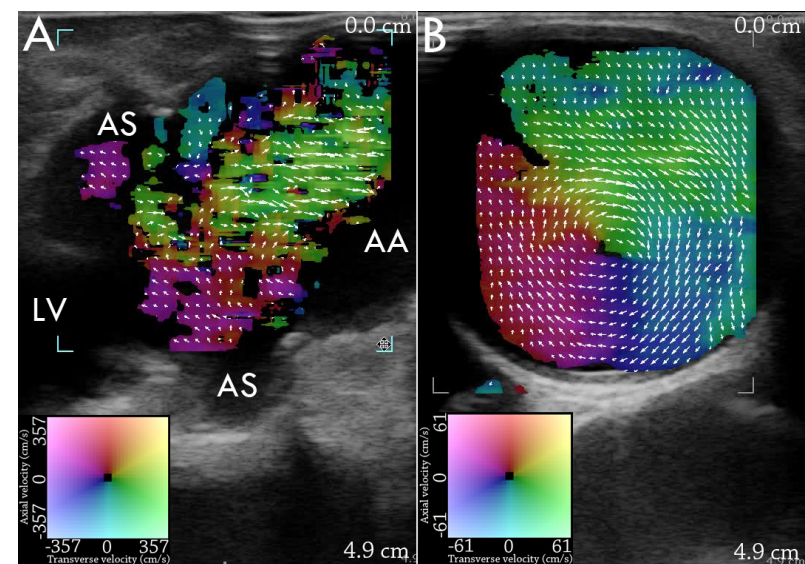

Fig. 11. Commercial TO vector flow image from the aorta. The arrows show the velocity direction and magnitude, which is also indicated by the colors. Image (A) shows the systolic flow in the ascending aorta in a patient with aortic stenosis. The flow has a mosaic pattern with a narrow jet. Image (B) shows secondary clock-wise flow in the ascending aorta of the same patients as in A. LV: left ventricle. AS: aortic sinus. AA: ascending aorta (From [156]).

correlation (0.91) between TO and MR data and a roughly $10 \%$ underestimation of the volume flow [45]. Another study used the same setup to compared MR with three ultrasound vector flow methods using TO, directional beamforming and synthetic aperture flow imaging. The study showed that the directional beamforming method has the least mean difference of the methods. These studies were conducted on the experimental RASMUS scanner [157]. Later studies were conducted on the commercial implementation on BK Ultrasound scanners [158] on for example vortices in the carotid artery [145],

\section{B. Volume flow in arteriovenous fistulas for hemodialysis}

An investigation of dialysis patients has been conducted [159]. Volume flow in arteriovenous fistulas (AVF) for hemodialysis was measured using TO Vector Flow Imaging with an UltraView 800 ultrasound scanner (BK Ultrasound, Herlev, Denmark). The results were compared to flow measurements using the current state-of-the-art ultrasound dilution technique (UDT) during dialysis. The initial study supported that "Vector Flow Imaging is applicable for volume flow measurements" [159].

A more comprehensive study investigated nineteen patients with matured functional AVF monthly over a six-month period comparing the UDT measurements with that from the UltraView 800 ultrasound scanner [160]. It was found that the TO volume flow measurements had a precision of $20 \%$ compared to the $30 \%$ precision of the UDT measurements. Also large change in volume flow between dialysis sessions detected by UDT was confirmed by VFI $(p=0.0001)$, but the concordance rate was poor $(0.72)$. The paper concluded that VFI is a reliable method for volume flow estimation and as is also a predictor of large volume flow changes over time in AVFs [160]. This points to the possibility of replacing the semi-invasive UDT measurements with a faster and more gentle external VFI examination.

\section{Grading carotid artery stenosis}

VFI was initially developed to eliminate the angle dependency of pulsed-wave or color Doppler [139], [161], the principal clinical applications being the assessment of carotid artery stenosis. Since a lumen narrowing leads to locally increased velocities, the peak systolic velocity (PSV) has logically become the most established criterion for stenosis grading [162], [163]. However, PSV measured by pulsed-wave Doppler has shown conflicting results with angiography. For this reason, ultrasonography alone is generally not accepted for clear-cut diagnosis [163]. Accuracy and precision are seriously affected by the errors in positioning the sample volume (at actual peak velocity location) and in accounting for the Doppler angle. Angle insonation larger than $60^{\circ}$ and precise position of the sample volume in the zone of greatest flow constriction (color flow mapping can help) are therefore recommended. PSV issues ought to be reduced in large part with real-time vector flow imaging [136]. It is expected that PSV measurement variability could be greatly reduced, especially when the carotid stenosis is tortuous [25]. The availability of clinical VFI for large vessels, such as in BK Ultrasound scanners [158], should facilitate such prospective studies. No clinical studies yet have been published to confirm the potential clinical benefit of ultrasound-based VFI when grading carotid artery stenosis using PSV.

\section{Left ventricular vortex formation and diastology}

An accurate assessment of the left ventricular function is of utmost clinical importance for the early detection of heart failure. Diagnosis of diastolic dysfunction can be very challenging, since history, physical examination, ECG, or chest $\mathrm{X}$-ray are often unhelpful. The wide accessibility of echocardiography and its ability to derive real-time non-invasive information make ultrasound the prerequisite technique for evaluating left ventricular diastolic function. However, current echocardiographic indices of diastolic function have major limitations that may hinder accurate diagnosis. Within the left ventricle of a normal heart, diastolic filling is characterized by the formation of an asymmetric swirling motion [164] that originates during early filling. In the normal heart, a large part of the left ventricular blood volume is actually involved in the vortex formation. Recent in-vivo observations suggest that the vortices that form during left ventricular filling have specific geometries and locations, which could be determinant factors of the diastolic function [42], [165], [114], [118], [166]. Intraventricular vortex flow imaging is becoming increasingly popular in the clinical literature and it is anticipated that accurate measures of the intracardiac vortex hemodynamics might play a key role in the assessment of the cardiac function (Fig. 8). So far the two clinical tools for ultrasound-based intracardiac vector flow imaging in adults are echo-PIV [41] and color-Doppler-based vector flow mapping [109], [60]. As mentioned above, these techniques are limited by their relatively low frame rates. Ongoing studies on transthoracic high-frame-rate echocardiography could change the situation in a near future [167], [122], [168]. 


\section{E. Left ventricular blood stasis}

Quantification of left ventricular blood stasis goes hand in hand with the above mentioned vortex formation. Left ventricular chamber dilation and depressed systolic function are known to be related to impaired filling, reduced ejection fraction, and increased occurrence of thromboembolism. Blood stasis in the heart chambers is a recognized risk factor for intracardiac thrombosis and cardiogenic thromboembolism. Using color-Doppler-based vector flow mapping (VFM) with a clinical GE Vivid scanner, Hendabadi et al. [117] and Rossini et al. [120] implemented an original Lagrangian method for mapping and quantifying blood stasis in the left ventricle. They observed that blood transport is altered in patients with dilated cardiomyopathy: an amount of blood is trapped by long-lasting vortices and needs several heart beats to leave the left ventricle. These studies confirm that a thorough analysis of the left ventricular hemodynamics is of clinical interest. This promising approach could be improved by the increased temporal resolution (using either VFM or speckle tracking) offered by high-frame-rate ultrasound imaging as described in the accompanying paper [1].

\section{F. VFI during open chest heart surgery}

Hansen et al. [170] studied the application of TO VFI during open chest heart surgery, where they could scan directly on the heart with a linear array probe during surgery. The method could in real time reveal new information about the complex flow in the heart.

Cardiac output measurements were investigated in a study with 25 patients undergoing cardiac bypass surgery [146]. They were scanned intraoperatively with TO on the ascending aorta and compared to transesophageal echocardiography (TEE) and pulmonary artery catheter thermodilution (PACTD). It was concluded that "TO with the present setup, is not interchangeable with PACTD for cardiac volume flow estimation, but is a reliable and precise angle-independent ultrasound alternative for velocity estimation of cardiac flow" [146]. A major reason is probably that the flow measured had a significant out-of-plane component, which biased the estimates, as flow rig measurements with the same equipment showed a precision between $5.5 \%$ and $14.7 \%$.

TO has also been used for scanning stenosed aortic valves for 20 patients with healthy valves, compared to 20 patients with diseased aortic valves before and after replacement [169]. Fig. 12 shows examples of flow for a normal valve (left image), diseased valve (middle) and after replacement (right). A clear improvement in the flow is seen with a fully laminar flow for the normal valve, highly disturbed flow for the diseased valve, and partly recovery for the replaced valve. The study points to the ability of making flow quantification during surgery to validate the correct replacement of a valve.

\section{G. Measurement of pressure gradients}

Non-invasive measurement of time-averaged pressure gradients is performed daily in echocardiographic laboratories for the assessment of valvular diseases. Time-resolved alongstreamline pressure gradients can also be estimated in the left ventricular chamber by adapting Euler's equation to Doppler velocities measured by color $\mathrm{M}$-mode. Intraventricular peak pressure gradients are potential early markers of the cardiac function [171], [172]. Two-dimensional relative pressure mapping derived from VFI was first described in the left ventricle using the Doppler-based VFM technique [173]. Comparisons with micromanometers were performed in an animal model. Recently, Olesen et al. determined longitudinal pressure gradients in a steady flow carotid model using the transverse oscillation method [174]. They then tested their technique with pulsatile flows [175]. Accurate estimation of relative pressures is still challenging. Turbulence, velocity measurement errors, mechanical hypotheses and boundary conditions all affect the 2-D pressure outputs. These studies, however, show that a robust time-resolved pressure estimation is likely to become possible with high-frame-rate vector flow imaging.

\section{H. Arterial flow patterns and wall shear rates}

It is known that arterial flow and wall shear rates affect atherosclerotic plaque formation and morphology [176]. Unlike conventional color Doppler, VFI allows deciphering the flow patterns in the large arteries. As discussed earlier, VFI can help to better grade carotid artery stenoses. Since full vector information is available, complex flow patterns can also be made visible and wall shear rates can be estimated. For example, Hansen et al. quantified the rotational flow and backflow occurring in the ascending aorta [177], a region susceptible to dilatation in patients with a bicuspid aortic valve [178]. Characterization of the rotational flow in the ascending aorta of such patients may help distinguish those at risk for development of ascending aortic aneurysm. Vector flow imaging also allows one to estimate wall shear rates in the carotid [16], [179], [180]. Although wall shear rates are known to play an important role in plaque development, the clinical impact of arterial vector flow imaging, however, has not yet been demonstrated. Detecting plaques with a high risk of rupture still remains a major challenge [181].

VFI can also give possibilities for new views of an organ than is currently possible due to beam-to-flow angles close to $90^{\circ}$. Brandt et al. [182] scanned the portal vein in the liver in subcostal and intercostal views and compared it to traditional spectral velocity estimation for finding the peak velocity. The scans were conducted on ten healthy volunteers over 3-5 heart beats. The two scan views gave similar results for TO VFI, but they were not consistent for the spectral estimates. This indicates that VFI can yield consistent scans from different directions, where traditional 1-D methods are limited to views with beam-to-flow angles less than $60^{\circ}$.

\section{Discussion - Challenges AND PERSPECTIVES}

Vector flow imaging has evolved from the first crossing beams systems in the late seventies to now being available on at least three commercial systems. The developments in digital systems, transducers, beamforming, advanced ultrasound fields, and dedicated estimators have made this possible and ultrasound can now provide real-time, quantitative flow data in a cross-sectional plane with an accuracy and precision 


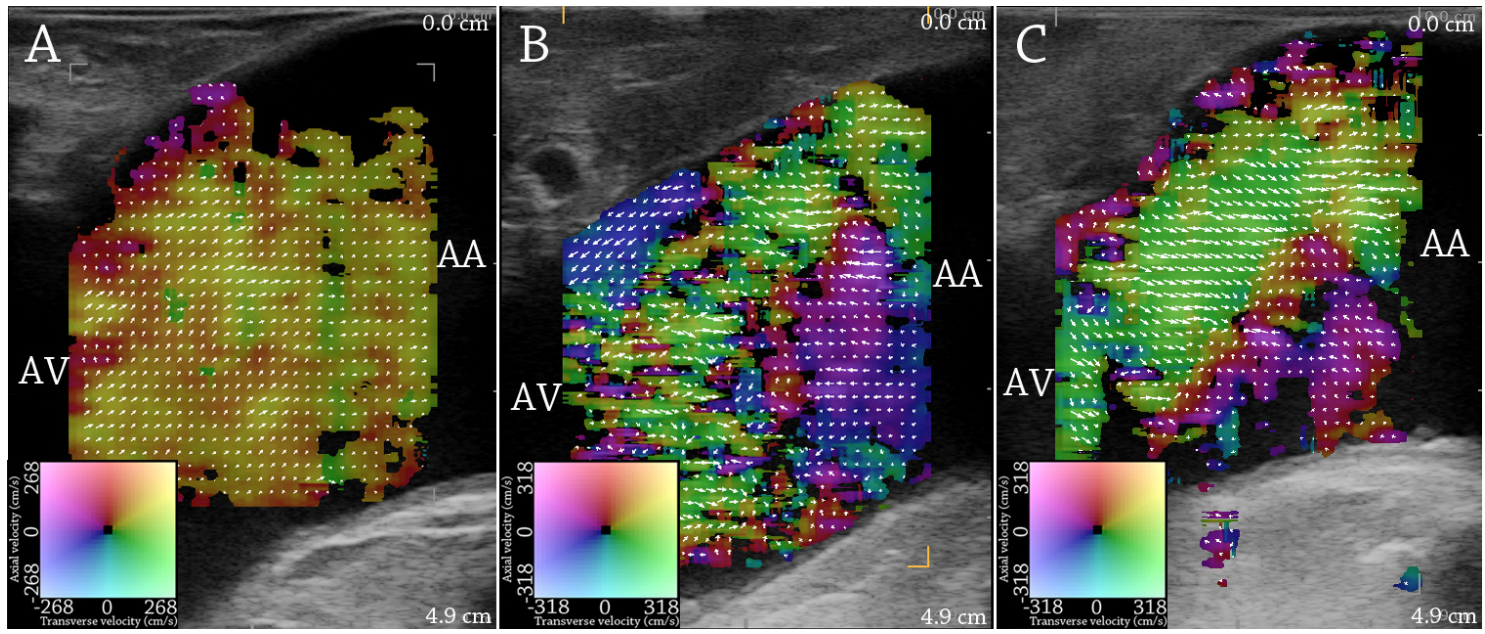

Fig. 12. Intraoperative TO estimation of the systolic blood flow in the ascending aorta in a patient with a normal aortic valve (A), and in a patient with a stenotic aortic valve before (B) and after valve replacement with a biologic prosthesis (C). AV: aortic valve; AA: ascending aorta. In (A) the arrows predominately points in the direction of the jet ejection, whereas a more chaotic flow is seen in (B). The direction is not fully restored in (C), but a clear improvement in the flow is seen after replacement (With permission from MD, PhD Kristoffer Lindskov Hansen, see also [169]).

better than $10 \%$. This expands the capabilities of ultrasound to better visualize the hemodynamics of patients, and will make quantification more correct and readily available. The amount of clinical studies are still very limited and restricted to smaller patient populations, and a real challenge is to expand the studies and demonstrate the efficacy of VFI and expand its use.

The display of VFI data is still fairly simple minded with arrows and underlying colors. The pulsating human flow is complex, where vortices appear in $100 \mathrm{~ms}$ and flow rapidly changes direction and magnitude. This complexity of the flow makes the display challenging, but also points to the wealth of information actually present in VFI.

VFI has been tested in a number of in-vivo situations, in the left ventricle, in the carotid artery, and for dialysis patients, whose external access is relatively easy. Heart valves have also been scanned during open-chest surgery. From a clinical viewpoint, quantification of the intraventricular flow is presently one of the more mature application of ultrasound-based VFI. This has been made possible by contrast-echo speckle tracking (echo-PIV) and color-Doppler-derived vector flow mapping. More studies have to be conducted on larger patient populations for VFI to become an accepted clinical standard, which is currently difficult due to its limited availability. VFI is readily available in only three scanners (BK Ultrasound, Hitachi, and Mindray) and can still be optimized in terms of clutter rejection, experimental validation, and reproducibility. To become a well-accepted clinical technique, VFI must prove to be less laboratory- and scanner-dependent than current 1-D methods. For years, ultrasound flow researchers were restricted to only one spatial dimension with color Doppler imaging. With ultrasound VFI, they have gained a second dimension.

The early clinical studies have also shown that the flow is not restricted to motion in the plane. Often a significant outof-plane velocity component is present and full quantification therefore demands a 3-D estimation. Adding the third spatial dimension was the next logical step. Four-dimensional (3-D + $t$ ) ultrasound vector flow imaging has emerged very recently and there is still a long way to go. It is very challenging due to the complicated transducers and many channels to handle in the beam steering in two directions. Early approaches have been developed and show promising results, but much is still to be done in terms of covering a full 3-D volume with velocity estimation at all points. The visualization is even more challenging and is still unresolved and inspiration should come from advanced methods of visualization developed for experimental fluid dynamics [183]. This comprehensive modality will surely benefit from the recent technical and clinical findings in 2-D VFI. In the future, it is likely that 4-D ultrasound vector flow imaging could become the modality of choice to analyze the blood flow non-invasively, mostly in the large arteries.

This paper has listed many of the methods developed for vector flow imaging. A good performance is attained for many of them, and the major challenge is probably not the estimation method but the limited amount of data available when using a traditional sequential acquisition. Only few emissions (816) are available, when a frame rate above $10-20 \mathrm{~Hz}$ must be attained [4], and often higher frame rates of 50 to $60 \mathrm{~Hz}$ are desired in e.g. cardiology. The few emissions make stationary echo canceling challenging, which also can be detrimental to the signal-to-noise ratio. Further, the limited data influences the attainable precision, as this is proportional to the number of observations entering the estimator. The largest improvement will therefore most likely come from parallel acquisition schemes, which are presented in the accompanying paper [1].

\section{ACKNOWLEDGEMENT}

This work was supported by grant 82-2012-4 from the Danish National Advanced Technology Foundation, by BK Ultrasound, Herlev, Denmark, and by discovery and accelerating grants of the Natural Sciences and Engineering Research Council of Canada (RPGIN-2016-04042, RGPIN-2015-04217 and RGPAS-477914-2015). 


\section{DISCLOSURE}

The authors have been involved in the development of many of the techniques presented in this review. Jørgen Arendt Jensen holds patents on TO VFI and directional VFI. He earns royalty from the selling of TO VFI systems by BK Ultrasound. Svetoslav Nikolov is employed by BK Ultrasound. Alfred C. $\mathrm{H}$. Yu has a provisional patent on vector projectile imaging that forms the basis of Mindray's V-Flow option.

\section{REFERENCES}

[1] J. A. Jensen, S. I. Nikolov, A. Yu, and D. Garcia, "Ultrasound vector flow imaging II: Parallel systems," IEEE Trans. Ultrason., Ferroelec., Freq. Contr., p. Accepted, 2016.

[2] D. H. Evans, W. N. McDicken, R. Skidmore, and J. P. Woodcock, Doppler Ultrasound, Physics, Instrumentation, and Clinical Applications. New York: John Wiley \& Sons, 1989.

[3] D. H. Evans and W. N. McDicken, Doppler Ultrasound, Physics, Instrumentation, and Signal Processing. New York: John Wiley \& Sons, 2000.

[4] J. A. Jensen, Estimation of Blood Velocities Using Ultrasound: A Signal Processing Approach. New York: Cambridge University Press, 1996.

[5] S. F. C. Stewart, "Effects of transducer, velocity, doppler angle, and instrument settings on the accuracy of color doppler ultrasound," Ultrasound Med. Biol., vol. 27, no. 4, pp. 551-564, 2001.

[6] B. Dunmire and K. W. Beach, "A Brief History of Vector Doppler," in Proc. SPIE Med. Imag., vol. 4325, Feb. 2001, pp. 200-214.

[7] G. E. Trahey, J. W. Allison, and O. T. von Ramm, "Angle independent ultrasonic detection of blood flow," IEEE Trans. Biomed. Eng., vol. BME-34, pp. 965-967, 1987.

[8] J. A. Jensen and P. Munk, "A new method for estimation of velocity vectors," IEEE Trans. Ultrason., Ferroelec., Freq. Contr., vol. 45, pp. 837-851, 1998.

[9] M. E. Anderson, "Multi-dimensional velocity estimation with ultrasound using spatial quadrature," IEEE Trans. Ultrason., Ferroelec., Freq. Contr., vol. 45, pp. 852-861, 1998.

[10] P. Peronneau, J.-P. Bournat, A. Bugnon, A. Barbet, and M. Xhaard, "Theoretical and practical aspects of pulsed doppler flowmetry realtime application to the measure of instantaneous velocity profiles in vitro and in vivo," in Cardiovascular applications of ultrasound R. Reneman, Ed. North Holland Publishing,, 1974, pp. 66-84.

[11] P. L. Hansen, G. Cross, and L. H. Light, "Beam-angle independent Doppler velocity measurement in superficial vessels," in Clinical Blood Flow Measurement. London: Sector Publishing, 1974.

[12] B. Dunmire, K. W. Beach, K.-H. Labs., M. Plett, and D. E. Strandness, "Cross-beam vector Doppler ultrasound for angle independent velocity measurements," Ultrasound Med. Biol., vol. 26, pp. 1213-1235, 2000.

[13] W. Q. Wang and L. X. Yao, "A double beam doppler ultrasound method for quantitative blood flow velocity measurement," Ultrasound Med. Biol., vol. 8, no. 4, pp. 421-425, 1982.

[14] A. Ashrafzadeh, J. Y. Cheung, and K. J. Dormer, "Analysis of velocity estimation error for a multidimensional doppler ultrasound system," IEEE Trans. Ultrason., Ferroelec., Freq. Contr., vol. 35, no. 5, pp. 536-544, 1988.

[15] S. Uematsu and S. Folstein, "Determination of volume of arterial blood flow by an ultrasonic device," Journal of Clinical Ultrasound, vol. 9, no. 5, pp. 209-216, 1981.

[16] P. Tortoli, G. Bambi, and S. Ricci, "Accurate Doppler angle estimation for vector flow measurements," IEEE Trans. Ultrason., Ferroelec., Freq. Contr., vol. 53, no. 8, pp. 1425-1431, Aug. 2006.

[17] S. Ricci, S. Diciotti, L. Francalanci, and P. Tortoli, "Accuracy and reproducibility of a novel dual-beam vector Doppler method," Ultrasound Med. Biol., vol. 35, no. 5, pp. 829-838, 2009.

[18] P. J. Phillips, A. P. Kadi, and O. T. von Ramm, "Feasibility study for a two-dimensional diagnostic ultrasound velocity mapping system," Ultrasound Med. Biol., vol. 21, no. 2, pp. 217-229, 1995.

[19] M. Scabia, M. Calzolai, L. Capineri, L. Masotti, and A. Fort, "A realtime two-dimensional pulsed-wave Doppler system," Ultrasound Med. Biol., vol. 26, no. 1, pp. 121-131, 2000.

[20] L. Capineri, M. Scabia, and L. Masotti, "A Doppler system for dynamic vector velocity maps," Ultrasound Med. Biol., vol. 28, no. 2, pp. 237 248,2002
[21] A. Pastorelli, G. Torricelli, M. Scabia, E. Biagi, and L. Masotti, "A realtime 2-D vector Doppler system for clinical experimentation," IEEE Trans. Med. Imag., vol. 27, no. 10, pp. 1515-1524, 2008.

[22] O. D. Kripfgans, J. M. Rubin, A. L. Hall, and J. B. Fowlkes, "Vector Doppler imaging of a spinning disc ultrasound Doppler phantom," Ultrasound Med. Biol., vol. 32, no. 7, pp. 1037-1046, 2006.

[23] A. L. Hall and R. B. Bernadi, "Method for detecting two-dimensional flow for ultrasound color flow imaging," US Patent 5398216; 1993.

[24] R. Steel and P. J. Fish, "Velocity bias and fluctuation in the standard dual beam Doppler reconstruction algorithm," IEEE Trans. Ultrason., Ferroelec., Freq. Contr., vol. 49, no. 10, pp. 1375-1383, 2002.

[25] R. Steel, K. V. Ramnarine, F. Davidson, P. J. Fish, and P. R. Hoskins, "Angle-independent estimation of maximum velocity through stenoses using vector Doppler ultrasound," Ultrasound Med. Biol., vol. 29, no. 4 , pp. 575-584, 2003.

[26] R. Steel, K. V. Ramnarine, A. Criton, F. Davidson, P. L. Allan, N. Humphries, H. F. Routh, P. J. Fish, and P. R. Hoskins, "Angledependence and reproducibility of dual-beam vector Doppler ultrasound in the common carotid arteries of normal volunteers," Ultrasound Med. Biol., vol. 30, no. 2, pp. 271-276, 2004.

[27] O. D. Kripfgans, J. M. Rubin, A. L. Hall, M. B. Gordon, and J. B. Fowlkes, "Measurement of volumetric flow," J. Ultrasound Med., vol. 25 , no. 10 , pp. 1305-1311, 2006.

[28] A. Swillens, P. Segers, H. Torp, and L. Løvstakken, "Two-dimensional blood velocity estimation with ultrasound: speckle tracking versus crossed-beam vector Doppler based on flow simulations in a carotid bifurcation model," IEEE Trans. Ultrason., Ferroelec., Freq. Contr., vol. 57, no. 2, pp. 327-339, 2010.

[29] A. Swillens, P. Segers, and L. Lovstakken, "Two-dimensional flow imaging in the carotid bifurcation using a combined speckle tracking and phase-shift estimator: a study based on ultrasound simulations and in vivo analysis," Ultrasound Med. Biol., vol. 36, no. 10, pp. 1722 $1735,2010$.

[30] M. D. Fox, "Multiple crossed-beam ultrasound Doppler velocimetry," IEEE Trans. Son. Ultrason., vol. SU-25, pp. 281-286, 1978.

[31] J. R. Overbeck, K. W. Beach, and D. E. Strandness, "Vector Doppler: Accurate measurement of blood velocity in two dimensions," Ultrasound Med. Biol., vol. 18, pp. 19-31, 1992.

[32] I. K. H. Tsang, B. Y. S. Yiu, and A. C. H. Yu, "A least-squares vector flow estimator for synthetic aperture imaging," in Proc. IEEE Ultrason. Symp., 2009, pp. 1387-1390.

[33] B. Y. Yiu, S. S. Lai, and A. C. Yu, "Vector projectile imaging: timeresolved dynamic visualization of complex flow patterns." Ultrasound Med. Biol., vol. 40, no. 9, pp. 2295-2309, sept 2014.

[34] H. K. H. So, J. Chen, B. Y. S. Yiu, and A. C. H. Yu, "Medical ultrasound imaging: to GPU or not to GPU?" IEEE Micro, vol. 31, no. 5, pp. 54-65, 2011.

[35] B. Y. S. Yiu, I. K. H. Tsang, and A. C. H. Yu, "GPU-based beamformer: Fast realization of plane wave compounding and synthetic aperture imaging," IEEE Trans. Ultrason., Ferroelec., Freq. Contr., vol. 58, no. 7, pp. 1698-1705, 2011.

[36] R. J. Adrian, "Particle-imaging techniques for experimental fluid mechanics," Annual Review of Fluid Mechanics, vol. 23, no. 1, pp. 261304, 1991.

[37] C. E. Willert and M. Gharib, "Digital particle image velocimetry," Exp. Fluids, vol. 10, no. 4, pp. 181-193, 1991.

[38] G. E. Trahey, S. M. Hubbard, and O. T. von Ramm, "Angle independent ultrasonic blood flow detection by frame-to-frame correlation of Bmode images," Ultrasonics, vol. 26, pp. 271-276, 1988.

[39] H. B. Kim, J. R. Hertzberg, and R. Shandas, "Development and validation of echo PIV," Exp. Fluids, vol. 36, pp. 455-462, 2004.

[40] H. Gao, N. Bijnens, D. Coisne, M. Lugiez, M. Rutten, and J. D'hooge, "2-D left ventricular flow estimation by combining speckle tracking with navier-stokes-based regularization: an in silico, in vitro and in vivo study," Ultrasound Med. Biol., vol. 41, no. 1, pp. 99-113, Jan 2015.

[41] P. P. Sengupta, B. K. Khandheria, J. Korinek, A. Jahangir, S. Yoshifuku, I. Milosevic, and M. Belohlavek, "Left ventricular isovolumic flow sequence during sinus and paced rhythms: new insights from use of highresolution doppler and ultrasonic digital particle imaging velocimetry," Journal of the American College of Cardiology, vol. 49, no. 8, pp. 899-908, 2007.

[42] G.-R. Hong, G. Pedrizzetti, G. Tonti, P. Li, Z. Wei, J. K. Kim, A. Baweja, S. Liu, N. Chung, H. Houle, and et al., "Characterization and quantification of vortex flow in the human left ventricle by contrast echocardiography using vector particle image velocimetry," JACC: Cardiovascular Imaging, vol. 1, no. 6, pp. 705-717, 2008. 
[43] A. Kheradvar, H. Houle, G. Pedrizzetti, G. Tonti, T. Belcik, M. Ashraf, J. R. Lindner, M. Gharib, and D. Sahn, "Echocardiographic particle image velocimetry: a novel technique for quantification of left ventricular blood vorticity pattern," Journal of the American Society of Echocardiography, vol. 23, no. 1, pp. 86-94, 2010.

[44] C. Poelma, R. M. E. van der Mijle, J. M. Mari, M. X. Tang, P. D. Weinberg, and J. Westerweel, "Ultrasound imaging velocimetry: toward reliable wall shear stress measurements," European Journal of Mechanics - B/Fluids, vol. 35, pp. 70-75, 2012.

[45] K. L. Hansen, J. Udesen, C. Thomsen, J. A. Jensen, and M. B. Nielsen, "In vivo validation of a blood vector velocity estimator with MR angiography," IEEE Trans. Ultrason., Ferroelec., Freq. Contr., vol. 56, no. 1, pp. 91-100, 2009.

[46] E. Konofagou and J. Ophir, "A new elastographic method for estimation and imaging of lateral displacements, lateral strains, corrected axial strains and poisson's ratios in tissues," Ultrasound Med. Biol., vol. 24, no. 8, pp. 1183-1199, 1998.

[47] J. Poree, D. Garcia, B. Chayer, J. Ohayon, and G. Cloutier, "Noninvasive vascular elastography with plane strain incompressibility assumption using ultrafast coherent compound plane wave imaging," IEEE Trans. Med. Imag., vol. 34, no. 12, pp. 2618-2631, 2015.

[48] I. A. Hein and W. D. O'Brien, "Current time-domain methods for assessing tissue motion by analysis from reflected ultrasound echoes - A review," IEEE Trans. Ultrason., Ferroelec., Freq. Contr., vol. 40, pp. 84-102, 1993.

[49] G. E. Mailloux, F. Langlois, P. Simard, and M. Bertrand, "Restoration of the velocity field of the heart from two-dimensional echocardiograms," IEEE Trans. Med. Imag., vol. 8, no. 2, pp. 143-153, Jun 1989.

[50] B. Friemel, L. Bohs, and G. Trahey, "Relative performance of twodimensional speckle-tracking techniques: normalized correlation, nonnormalized correlation and sum-absolute-difference," in Proc. IEEE Ultrason. Symp., 1995, pp. 1481-1484.

[51] F. Yeung, S. F. Levinson, and K. J. Parker, "Multilevel and motion model-based ultrasonic speckle tracking algorithms," Ultrasound Med. Biol., vol. 24, no. 3, pp. 427-441, 1998.

[52] I. Cespedes, Y. Huang, J. Ophir, and S. Spratt, "Methods for estimation of subsample time delays of digitized echo signals," Ultrasonic Imaging, vol. 17, no. 2, pp. 142-171, 1995, pMID: 7571208.

[53] B. Pan, K. Qian, H. Xie, and A. Asundi, "Two-dimensional digital image correlation for in-plane displacement and strain measurement: a review," Measurement Science and Technology, vol. 20, no. 6, p. 062001, 2009.

[54] F. Zhang, C. Lanning, L. Mazzaro, A. J. Barker, P. E. Gates, W. D Strain, J. Fulford, O. E. Gosling, A. C. Shore, N. G. Bellenger, and et al., "In vitro and preliminary in vivo validation of echo particle image velocimetry in carotid vascular imaging," Ultrasound Med. Biol., vol. 37, no. 3, pp. 450-464, 2011.

[55] S. Cimino, G. Pedrizzetti, G. Tonti, E. Canali, V. Petronilli, L. D. Luca, C. Iacoboni, and L. Agati, "In vivo analysis of intraventricular fluid dynamics in healthy hearts," European Journal of Mechanics B/Fluids, vol. 35, pp. 40-46, 2012.

[56] M. Raffel, C. E. Willert., S. Wereley, and J. Kompenhans, Particle image velocimetry: a practical guide, 2nd ed. Springer, 2007.

[57] D. Garcia, "A fast all-in-one method for automated post-processing of piv data," Experiments in Fluids, vol. 50, no. 5, pp. 1247-1259, Oct 2010.

[58] A. Vlasenko and C. Schnorr, "Physically consistent and efficient variational denoising of image fluid flow estimates," IEEE Transactions on Image Processing, vol. 19, no. 3, pp. 586-595, 2010.

[59] H. Abe, G. Caracciolo, A. Kheradvar, G. Pedrizzetti, B. K. Khandheria, J. Narula, and P. P. Sengupta, "Contrast echocardiography for assessing left ventricular vortex strength in heart failure: a prospective cohort study," European Heart Journal - Cardiovascular Imaging, vol. 14, no. 11, pp. 1049-1060, Nov 2013.

[60] P. P. Sengupta, G. Pedrizzetti, P. J. Kilner, A. Kheradvar, T. Ebbers, G. Tonti, A. G. Fraser, and J. Narula, "Emerging trends in CV flow visualization," JACC: Cardiovascular Imaging, vol. 5, no. 3, pp. 305316,2012

[61] S. Fadnes, S. A. Nyrnes, H. Torp, and L. Lovstakken, "Shunt flow evaluation in congenital heart disease based on two-dimensional speckle tracking," Ultrasound Med. Biol., vol. 40, no. 10, pp. 2379-2391, 2014.

[62] P. Munk, "Estimation of the 2-D flow vector in ultrasonic imaging: A new approach," Master's thesis, Department of Information Technology, Technical University of Denmark, 1996.

[63] J. A. Jensen, "Apparatus and method for determining movements and velocities of moving objects," International patent PCT/DK97/00287, 1996.
[64] M. E. Anderson, "Spatial quadrature: a novel technique for multidimensional velocity estimation," in Proc. IEEE Ultrason. Symp., vol. 45, 1997, pp. 1233-1238.

[65] J. W. Goodman, Introduction to Fourier optics, 2nd ed. New York: McGraw Hill Inc., 1996.

[66] J. A. Jensen and P. Munk, "An improved estimation and focusing scheme for vector velocity estimation," in Proc. IEEE Ultrason. Symp., vol. 2, 1999, pp. 1465-1470.

[67] H. Liebgott, J. Wilhjelm, J. A. Jensen, D. Vray, and P. Delachartre, "PSF dedicated to estimation of displacement vectors for tissue elasticity imaging with ultrasound," IEEE Trans. Ultrason., Ferroelec., Freq. Contr, vol. 54, no. 4, pp. 746-756, 2007.

[68] R. F. Kerr and M. E. Anderson, "Velocity envelope of vector flow estimation with spatial quadrature," in SPIE Medical Imaging, vol. 5035, 2003, pp. 265-276.

[69] H. Liebgott, A. Basarab, P. Gueth, C. Cachard, and P. Delachartre, "Lateral RF image synthesis using a synthetic aperture imaging technique." IEEE Trans. Ultrason., Ferroelec., Freq. Contr., vol. 55, no. 9 , pp. 2097-2103, 2008.

[70] M. Lenge, A. Ramalli, A. Cellai, P. Tortoli, C. Cachard, and H. Liebgott, "A new method for 2d-vector blood flow imaging based on unconventional beamforming techniques," in IEEE International Conference on Acoustics, Speech and Signal Processing (ICASSP), May 2014, pp. $5125-5129$.

[71] J. A. Jensen, A. H. Brandt, and M. B. Nielsen, "Convex array vector velocity imaging using transverse oscillation and its optimization," IEEE Trans. Ultrason., Ferroelec., Freq. Contr., vol. 62, no. 12, pp. 2043-2053, 2015.

[72] J. A. Jensen, "Improved vector velocity estimation using directional transverse oscillation," in Proc. IEEE Ultrason. Symp., October 2015 , pp. $1-4$.

[73] _ "Directional transverse oscillation vector flow estimation," IEEE Trans. Ultrason., Ferroelec., Freq. Contr., vol. 63, p. Submitted, 2016.

[74] C. Kasai, K. Namekawa, A. Koyano, and R. Omoto, "Real-Time Two-Dimensional Blood Flow Imaging using an Autocorrelation Technique," IEEE Trans. Son. Ultrason., vol. 32, pp. 458-463, 1985.

[75] M. E. Anderson, "A heterodyning demodulation technique for spatial quadrature," in Proc. SPIE Med. Imag., 2000, pp. 1487-1490.

[76] J. A. Jensen, "A new estimator for vector velocity estimation," IEEE Trans. Ultrason., Ferroelec., Freq. Contr., vol. 48, no. 4, pp. 886-894, 2001.

[77] J. Udesen and J. A. Jensen, "Investigation of Transverse Oscillation Method," IEEE Trans. Ultrason., Ferroelec., Freq. Contr., vol. 53, pp. 959-971, 2006.

[78] A. Basarab, P. Gueth, H. Liebgott, and P. Delachartre, "Phase-based block matching applied to motion estimation with unconventional beamforming strategies," IEEE Trans. Ultrason., Ferroelec., Freq. Contr., vol. 56, no. 5, pp. 945-957, 2009.

[79] C. Sumi, "Displacement vector measurement using instantaneous ultrasound signal phase - multidimensional autocorrelation and Doppler methods," IEEE Trans. Ultrason., Ferroelec., Freq. Contr., vol. 55, no. 1 , pp. $24-43,2008$.

[80] M. E. Anderson, "Vector flow estimator isomorphism and wall filter requirements," in Proc. SPIE Med. Imag., vol. 4325, 2001, pp. 215226.

[81] J. Udesen, M. B. Nielsen, K. R. Nielsen, and J. A. Jensen, "Examples of in-vivo blood vector velocity estimation," Ultrasound Med. Biol., vol. 33, pp. 541-548, 2007.

[82] M. J. Pihl, J. Marcher, and J. A. Jensen, "Phased-array vector velocity estimation using transverse oscillations," IEEE Trans. Ultrason., Ferroelec., Freq. Contr., vol. 59, no. 12, pp. 2662-2675, 2012.

[83] M. J. Pihl and J. A. Jensen, "A transverse oscillation approach for estimation of three-dimensional velocity vectors. Part I: Concept and simulation study," IEEE Trans. Ultrason., Ferroelec., Freq. Contr., vol. 61, pp. 1599-1607, 2014.

[84] M. J. Pihl, M. B. Stuart, B. G. Tomov, M. F. Rasmussen, and J. A. Jensen, "A transverse oscillation approach for estimation of threedimensional velocity vectors. Part II: Experimental validation," IEEE Trans. Ultrason., Ferroelec., Freq. Contr., vol. 51, no. 10, pp. 16081618, 2014.

[85] D. Dotti, E. Gatti, V. Svelto, A. Uggè, and P. Vidali, "Blood flow measurements by ultrasound correlation techniques," Energia Nucleare, vol. 23, pp. 571-575, 1976.

[86] M. Bassini, D. Dotti, E. Gatti, T. Longo, G. Martinis, P. Pignoli, and P. Pizzolati, "An ultrasonic non-invasive blood flow-meter based on cross-correlation techniques," in Ultrason Int Proc, 1979, pp. 273-278. 
[87] M. Bassini, E. Gatti, T. Longo, G. Martinis, P. Pignoli, and P. L. Pizzolati, "In vivo recording of blood velocity profiles and studies in vitro of profile alterations induced by known stenoses," Texas Heart Inst. J., vol. 9, pp. 185-194, 1982.

[88] O. Bonnefous, P. Pesqué, and X. Bernard, "A new velocity estimator for color flow mapping," in Proc. IEEE Ultrason. Symp., 1986, pp. 855-860.

[89] S. G. Foster, "A pulsed ultrasonic flowmeter employing time domain methods," Ph.D. dissertation, Dept. Elec. Eng., University of Illinois, Urbana, Ill., 1985.

[90] S. G. Foster, P. M. Embree, and W. D. O’Brien, "Flow velocity profile via time-domain correlation: Error analysis and computer simulation," IEEE Trans. Ultrason., Ferroelec., Freq. Contr., vol. 37, pp. 164-175, 1990.

[91] O. Bonnefous, "Measurement of the complete (3D) velocity vector of blood flows," in Proc. IEEE Ultrason. Symp., 1988, pp. 795-799.

[92] J. A. Jensen, "Directional velocity estimation using focusing along the flow direction: I: Theory and simulation," IEEE Trans. Ultrason., Ferroelec., Freq. Contr., vol. 50, pp. 857-872, 2003.

[93] J. A. Jensen and R. Bjerngaard, "Directional velocity estimation using focusing along the flow direction: II: Experimental investigation," IEEE Trans. Ultrason., Ferroelec., Freq. Contr., vol. 50, pp. 873-880, 2003.

[94] J. A. Jensen and S. I. Nikolov, "Directional synthetic aperture flow imaging," IEEE Trans. Ultrason., Ferroelec., Freq. Contr., vol. 51, pp. 1107-1118, 2004.

[95] J. A. Jensen, "Comparison of vector velocity imaging using directional beamforming and transverse oscillation for a convex array transducer," in Proceedings of the SPIE - Progress in Biomedical Optics and Imaging, vol. 9040. SPIE - International Society for Optical Engineering, 2014, pp. $904012-1-8$.

[96] J. Kortbek and J. A. Jensen, "Estimation of velocity vector angles using the directional cross-correlation method," IEEE Trans. Ultrason., Ferroelec., Freq. Contr., vol. 53, pp. 2036-2049, 2006.

[97] A. R. Walker, D. J. Phillips, and J. E. Powers, "Evaluating Doppler devices using a moving string test target," J. Clinical Ultrasound, vol. 10 , no. 1 , pp. 25-30, 1982

[98] V. L. Newhouse, L. W. Varner, and P. J. Bendick, "Geometrical spectrum broadening in ultrasonic Doppler systems," IEEE Trans. Biomed. Eng., vol. BME-24, pp. 478-480, 1977.

[99] V. L. Newhouse, A. R. Ehrenwald, and G. F. Johnson, "The effect of Rayleigh scattering and frequency dependent absorption on the output spectrum of Doppler blood flowmeters," Ultrasound Med., vol. 3B, pp. 1181-1191, 1977.

[100] V. L. Newhouse, E. S. Furgason, G. F. Johnson, and D. A. Wolf, "The dependence of ultrasound Doppler bandwidth on beam geometry," IEEE Trans. Son. Ultrason., vol. SU-27, pp. 50-59, 1980.

[101] V. L. Newhouse, D. Censor, T. Vontz, J. A. Cisneros, and B. B. Goldberg, "Ultrasound Doppler probing of flows transverse with respect to beam axis," IEEE Trans. Biomed. Eng., vol. BME-34, pp. 779-788, 1987.

[102] V. L. Newhouse, K. S. Dickerson, D. Cathignol, and J.-Y. Chapelon, "Three-dimensional vector flow estimation using two transducers and spectral width," IEEE Trans. Ultrason., Ferroelec., Freq. Contr., vol. 41, pp. 90-95, 1994

[103] P. Tortoli, G. Guidi, and P. Pignoli, "Transvers Doppler spectral analysis for a correct interpretation of flow sonograms," Ultrasound Med. Biol., vol. 19, pp. 115-121, 1993.

[104] P. Tortoli, G. Guidi, F. Guidi, and C. Atzeni, "A review of experimental transverse Doppler studies," IEEE Trans. Ultrason., Ferroelec., Freq. Contr., vol. 41, pp. 84-89, 1994.

[105] C. A. C. Bastos, P. J. Fish, and F. Vaz, "Spectrum of Doppler ultrasound signals from nonstationary blood flow," IEEE Trans. Ultrason., Ferroelec., Freq. Contr., vol. 46, no. 5, pp. 1201-1217, 1999.

[106] P. Tortoli, A. Dallai, E. Boni, L. Francalanci, and S. Ricci, "An automatic angle tracking procedure for feasible vector Doppler blood velocity measurements," Ultrasound Med. Biol., vol. 36, no. 3, pp. 488-496, 2010.

[107] B.-F. Osmanski, G. Montaldo, and M. Tanter, "Out-of-plane doppler imaging based on ultrafast plane wave imaging," IEEE Trans. Ultrason., Ferroelec., Freq. Contr., vol. 62, no. 4, pp. 625-636, April 2015.

[108] J. A. Jensen, "Transverse spectral velocity estimation," IEEE Trans. Ultrason., Ferroelec., Freq. Contr., vol. 61, no. 11, pp. 1815-1823, november 2014.

[109] D. Garcia, J. C. del Alamo, D. Tanne, R. Yotti, C. Cortina, E. Bertrand, J. C. Antoranz, E. Perez-David, R. Rieu, F. Fernandez-Aviles, and J. Bermejo, "Two-dimensional intraventricular flow mapping by dig- ital processing conventional color-doppler echocardiography images," IEEE Trans. Med. Imag., vol. 29, no. 10, pp. 1701-1713, 2010

[110] F. Mehregan, F. Tournoux, S. Muth, P. Pibarot, R. Rieu, G. Cloutier, and D. Garcia, "Doppler vortography: a color doppler approach for quantification of the intraventricular blood flow vortices," Ultrasound Med. Biol., vol. 40, no. 1, pp. 210-221, January 2014.

[111] S. Ohtsuki and M. Tanaka, "The flow velocity distribution from the doppler information on a plane in three-dimensional flow," $J$. of Visualization, vol. 9, no. 1, pp. 69-82, 2006.

[112] T. Uejima, A. Koike, H. Sawada, T. Aizaw, S. Ohtsuki, M. Tanaka, T. Furukawa, and A. G. Fraser, "A new echocardiographic method for identifying vortex flow in the left ventricle: Numerical validation," Ultrasound Med. Biol., vol. 36, no. 5, pp. 772 - 788, 2010.

[113] T. Tanaka, R. Asami, K. Kawabata, E. K. Itatani, T. Uejima, T. Nishiyama, and T. Okada, "Intracardiac vfm technique using diagnostic ultrasound system," Hitachi Review, vol. 64, no. 8, p. 489 , 2015.

[114] Y. Nogami, T. Ishizu, A. Atsumi, M. Yamamoto, R. Kawamura, Y. Seo, and K. Aonuma, "Abnormal early diastolic intraventricular flow 'kinetic energy index' assessed by vector flow mapping in patients with elevated filling pressure," European Heart Journal - Cardiovascular Imaging, vol. 14, no. 3, pp. 253-260, 2013, pMID: 22822154.

[115] R. Ro, D. Halpern, D. J. Sahn, P. Homel, M. Arabadjian, C. Lopresto, and M. V. Sherrid, "Vector flow mapping in obstructive hypertrophic cardiomyopathy to assess the relationship of early systolic left ventricular flow and the mitral valve," Journal of the American College of Cardiology, vol. 64, no. 19, pp. 1984-1995, 2014.

[116] M. Stugaard, H. Koriyama, K. Katsuki, K. Masuda, T. Asanuma, Y. Takeda, Y. Sakata, K. Itatani, and S. Nakatani, "Energy loss in the left ventricle obtained by vector flow mapping as a new quantitative measure of severity of aortic regurgitation: a combined experimental and clinical study," Eur Heart J Cardiovasc Imaging, vol. 16, no. 7, pp. 723-730, 2015, pMID: 25762562 .

[117] S. Hendabadi, J. Bermejo, Y. Benito, R. Yotti, F. Feraindez-Avilas, J. C. del Alamo, and S. C. Shadden, "Topology of blood transport in the human left ventricle by novel processing of doppler echocardiography," Annals of Biomedical Engineering, vol. 41, no. 12, pp. 2603-2616, 2013.

[118] J. Bermejo, Y. Benito, M. Alhama, R. Yotti, P. Martanez-Legazpi, C. P. del Villar, E. Parez-David, A. Gonzailez-Mansilla, C. S.-M Barrio, and et al., "Intraventricular vortex properties in nonischemic dilated cardiomyopathy," American Journal of Physiology - Heart and Circulatory Physiology, vol. 306, no. 5, pp. 718-729, 2014, pMID: 24414062.

[119] J. Bermejo, P. Martanez-Legazpi, and J. C. del Alamo, "The clinical assessment of intraventricular flows," Annual Review of Fluid Mechanics, vol. 47, no. 1, pp. 315-342, 2015.

[120] L. Rossini, P. Martinez-Legazpi, V. V. andL. Fernandez-Friera, C. P. P. del Villar, S. Rodriguez-Lopez, Y. Benito, M.-G. Borja, D. PastorEscuredo, R. Yotti, and et al., "A clinical method for mapping and quantifying blood stasis in the left ventricle," Journal of Biomechanics, p. In press, 2016.

[121] O. Bonnefous and P. Pesqué, "Time domain formulation of pulseDoppler ultrasound and blood velocity estimation by cross correlation," Ultrason. Imaging, vol. 8, pp. 73-85, 1986.

[122] D. Posada, J. Poree, A. Pellissier, B. Chayer, F. Tournoux, G.Cloutier, and D. Garcia, "Staggered multiple-prf ultrafast color Doppler," IEEE Trans. Med. Imag., vol. PP, no. 99, p. Online, 2016.

[123] M. D. Fox and W. M. Gardiner, "Three-dimensional Doppler velocimetry of flow jets," IEEE Trans. Biomed. Eng., vol. 35, pp. 834-841, 1988.

[124] A. Gomez, K. Pushparajah, J. M. Simpson, D. Giese, T. Schaeffter, and G. Penney, "A sensitivity analysis on $3 \mathrm{~d}$ velocity reconstruction from multiple registered echo doppler views," Med. Image Anal., vol. 17, no. 6 , pp. 616-631, 2013.

[125] I. A. Hein, "Triple-beam lens transducers for three-dimensional ultrasonic fluid flow estimation," IEEE Trans. Ultrason., Ferroelec., Freq. Contr., vol. 42, pp. 854-869, 1995.

[126] J. Meunier, "Tissue motion assessment from 3D echographic speckle tracking," Phys. Med. Biol., vol. 43, pp. 1241-1254, 1998.

[127] J. Crosby, B. H. Amundsen, T. Hergum, E. W. Remme, S. Langeland, and H. Torp, "3-d speckle tracking for assessment of regional left ventricular function," Ultrasound Med. Biol., vol. 35, no. 3, pp. 458471, 2009

[128] C. Jia, K. Kim, T. J. Kolias, J. M. Rubin, W. F. Weitzel, P. Yan, D. Dione, A. J. Sinusas, J. Duncan, and M. O'Donnell, " 4 d elasticity imaging of pva lv phantom integrated with pulsatile circulation system 
using 2d phased array," Proc. IEEE Ultrason. Symp., pp. 876-879, 2007.

[129] M. J. Pihl and J. A. Jensen, "Measuring 3D velocity vectors using the transverse oscillation method," in Proc. IEEE Ultrason. Symp., 2012, pp. 1881-1885.

[130] M. J. Pihl, M. B. Stuart, B. G. Tomov, J. M. Hansen, M. F. Rasmussen, and J. A. Jensen, "Preliminary examples of 3D vector flow imaging," in Proc. SPIE Med. Imag., vol. 8675, 2013, pp. 86750H-1-86750H-12.

[131] T. A. Maniatis, R. S. C. Cobbold, and K. W. Johnston, "Twodimensional velocity reconstruction strategies for color flow Doppler ultrasound images," Ultrasound Med. Biol., vol. 20, pp. 137-145, 1994.

[132] — "Flow imaging in an end-to-side anastomosis model using twodimensional velocity vectors," Ultrasound Med. Biol., vol. 20, no. 6, pp. 559-569, 1994.

[133] I. K. Ekroll, A. Swillens, P. Segers, T. Dahl, H. Torp, and L. Løvstakken, "Simultaneous quantification of flow and tissue velocities based on multi-angle plane wave imaging," IEEE Trans. Ultrason., Ferroelec., Freq. Contr., vol. 60, no. 4, pp. 727-738, 2013

[134] J. Udesen, F. Gran, K. L. Hansen, J. A. Jensen, C. Thomsen, and M. B. Nielsen, "High frame-rate blood vector velocity imaging using plane waves: simulations and preliminary experiments," IEEE Trans. Ultrason., Ferroelec., Freq. Contr., vol. 55, no. 8, pp. 1729-1743, 2008.

[135] S. Fadnes, I. K. Ekroll, S. A. Nyrnes, H. Torp, and L. Løvstakken, "Robust angle-independent blood velocity estimation based on dualangle plane wave imaging," IEEE Trans. Ultrason., Ferroelec., Freq. Contr., vol. 62, no. 10, pp. 1757-1767, October 2015.

[136] S. Ricci, L. Bassi, and P. Tortoli, "Real-time vector velocity assessment through multigate Doppler and plane waves," IEEE Trans. Ultrason., Ferroelec., Freq. Contr., vol. 61, no. 2, pp. 314-324, 2014

[137] L. N. Bohs and G. E. Trahey, "A novel method for angle independent ultrasonic imaging of blood flow and tissue motion," IEEE Trans. Biomed. Eng., vol. 38, pp. 280-286, 1991.

[138] N. Vera, D. A. Steinman, C. R. Ethier, K. W. Johnston, and R. S. C. Cobbold, "Visualization of complex flow field, with application to the interpretation of colour flow Doppler images," Ultrasound Med. Biol., vol. 18, pp. 1-9, 1992.

[139] D. Y. Fei, C. T. Fu, W. H. Brewer, and K. A. Kraft, "Angle independent Doppler color imaging: Determination of accuracy and a method of display," Ultrasound Med. Biol., vol. 20, no. 2, pp. 147-155, 1994.

[140] D. Y. Fei and C. T. Fu, "New method to obtain ultrasonic angle independent Doppler color images using a sector transducer," Annals of Biomedical Engineering, vol. 27, no. 2, pp. 187-193, 1999.

[141] J. Flynn, R. Daigle, L. Pflugrath, and P. Kaczkowski, "High frame rate vector velocity blood flow imaging using a single plane wave transmission angle," in Proc. IEEE Ultrason. Symp., 2012, pp. 323325.

[142] D. H. Evans, J. A. Jensen, and M. B. Nielsen, "Ultrasonic colour Doppler imaging," Interface Focus, vol. 1, no. 4, pp. 490-502, 2011.

[143] M. M. Pedersen, M. J. Pihl, P. Haugaard, J. M. Hansen, K. L. Hansen, M. B. Nielsen, and J. A. Jensen, "Comparison of real-time in vivo spectral and vector velocity estimation," Ultrasound Med. Biol., vol. 38 , no. 1, pp. 145-151, 2012.

[144] P. Haugaard and G. Seerup, "Vector flow ultrasound imaging," 2012, uS Patent Application 13/613,099;

[145] M. M. Pedersen, M. J. Pihl, P. Haugaard, K. L. Hansen, T. Lange, L. Lonn, M. B. Nielsen, and J. A. Jensen, "Novel flow quantification of the carotid bulb and the common carotid artery with vector flow ultrasound," Ultrasound Med. Biol., vol. 40, no. 11, pp. 2700-2706, 2014.

[146] K. L. Hansen, H. Møller-Sørensen, J. Kjaergaard, M. B. Jensen, J. T. Lund, M. M. Pedersen, J. B. Olesen, J. A. Jensen, and M. B. Nielsen, "Vector flow imaging compared with conventional doppler ultrasound and thermodilution for estimation of blood flow in the ascending aorta," Ultrasonic Imaging, pp. 1-16, 2015.

[147] D. A. H. Steinman and D. A. Steinman, "The art and science of visualizing simulated blood-flow dynamics," Leonardo, vol. 40, no. 1, pp. 71-76, 2007.

[148] T. McLoughlin, R. S. Laramee, R. Peikert, F. H. Post, and M. Chen, "Over two decades of integration-based, geometric flow visualization," Computer Graphics Forum, vol. 29, no. 6, pp. 1807-1829, 2010.

[149] J. Flynn, R. Daigle, L. Pflugrath, P. Kaczkowski, and K. Linkhart, "Estimation and display for vector Doppler imaging using planewave transmissions," Proc. IEEE Ultrason. Symp., pp. 413-418, 2011.

[150] B. Y. S. Yiu and A. C. H. Yu, "High-frame-rate ultrasound colorencoded speckle imaging of complex flow dynamics," Ultrasound Med. Biol., vol. 39, no. 6, pp. 1015-1025, 2013.
[151] A. C. H. Yu and Y. S. Yiu, "Apparatus for ultrasound flow vector imaging and methods thereof," 2014, patent Application PCT/CN2014/091035;

[152] S. Shahriari and D. Garcia, "A particle-based simulation tool for ultrasound blood flow imaging: validation of high-speed echo-piv," in Proc. IEEE Ultrason. Symp., 2013, pp. 2034-2037.

[153] M. M. Pedersen, M. J. Pihl, J. M. Hansen, P. M. Hansen, P. Haugaard, M. B. Nielsen, and J. A. Jensen, "Secondary arterial blood flow patterns visualised with vector flow ultrasound," in Proc. IEEE Ultrason. Symp. 2011, pp. 1242-1245.

[154] P. Angelelli, S. R. Snare, S. A. Nyrnes, S. Bruckner, H. Hauser, and L. Løvstakken, "Live ultrasound-based particle visualization of blood flow in the heart," in Proceedings of the 30th Spring Conference on Computer Graphics, 2014, pp. 42-49.

[155] G. Haller, "Lagrangian coherent structures," Annu. Rev. Fluid Mech, vol. 47, pp. 137-162, 2015.

[156] K. L. Hansen, H. Møller-Sørensen, M. M. Pedersen, P. M. Hansen, J. Kjaergaard, J. T. Lund, J. C. Nilsson, J. A. Jensen, and M. B. Nielsen, "First report on intraoperative vector flow imaging of the heart among patients with healthy and diseased aortic valves," Ultrasonics, vol. 56 , pp. 243-250, 2014.

[157] J. A. Jensen, O. Holm, L. J. Jensen, H. Bendsen, S. I. Nikolov, B. G. Tomov, P. Munk, M. Hansen, K. Salomonsen, J. Hansen, K. Gormsen, H. M. Pedersen, and K. L. Gammelmark, "Ultrasound research scanner for real-time synthetic aperture image acquisition," IEEE Trans. Ultrason., Ferroelec., Freq. Contr., vol. 52 (5), pp. 881891, May 2005.

[158] P. M. Hansen, M. M. Pedersen, K. L. Hansen, M. B. Nielsen, and J. A. Jensen, "Demonstration of a vector velocity technique," Ultraschall in Med., vol. 32, pp. 213-215, 2011.

[159] P. M. Hansen, J. B. Olesen, M. J. Pihl, T. Lange, S. Heerwagen, M. M. Pedersen, M. Rix, L. Lønn, J. A. Jensen, and M. B. Nielsen, "Volume flow in arteriovenous fistulas using vector velocity ultrasound," Ultrasound Med. Biol., vol. 40, no. 11, pp. 2707-2714, 2014.

[160] A. H. Brandt, J. Jensen, K. L. Hansen, P. M. Hansen, T. Lange, M. Rix, J. A. Jensen, L. Lönn, and M. B. Nielsen, "Surveillance for hemodialysis access stenosis: Usefulness of ultrasound vector volume flow," Eur Radiol, p. Under review, 2016.

[161] P. R. Hoskins, "Peak velocity estimation in arterial stenosis models using colour vector doppler," Ultrasound Med. Biol., vol. 23, no. 6, pp. 889-897, 1997.

[162] E. G. Grant, C. B. Benson, and G. L. M. et al, "Carotid artery stenosis: Gray-scale and Doppler US diagnosis - society of radiologists in ultrasound consensus conference," Radiology, vol. 229, no. 2, pp. 340-346, 2003.

[163] G. M. von Reutern, M. W. Goertler, N. M. Bornstein, M. D. Sette, D. H. Evans, A. Hetzel, M. Kaps, F. Perren, and et al., "Grading carotid stenosis using ultrasonic methods," Stroke, vol. 43, no. 3, pp. 916-921, 2012.

[164] P. J. Kilner, G.-Z. Yang, A. J. Wilkes, R. H. Mohiaddin, D. N. Firmin, and M. H. Yacoub, "Asymmetric redirection of flow through the heart," Nature, vol. 404, no. 6779, pp. 759-761, Apr 2000.

[165] G. Nucifora, V. Delgado, M. Bertini, N. A. Marsan, N. R. V. de Veire A. C. T. Ng, H.-M. J. Siebelink, M. J. Schalij, E. R. Holman, P. P Sengupta, and et al., "Left ventricular muscle and fluid mechanics in acute myocardial infarction," The American Journal of Cardiology, vol. 106, no. 10, pp. 1404-1409, 2010.

[166] P. Martinez-Legazpi, J. Bermejo, Y. Benito, R. Yotti, C. P. del Villar, A. Gonzalez-Mansilla, A. Barrio, E. Villacorta, P. L. Sanchez, F. Fernaqndez-Aviles, and et al., "Contribution of the diastolic vortex ring to left ventricular filling," Journal of the American College of Cardiology, vol. 64, no. 16, pp. 1711-1721, 2014.

[167] C. Papadacci, M. Pernot, M. Couade, M. Fink, and M. Tanter, "Highcontrast ultrafast imaging of the heart," IEEE Trans. Ultrason., Ferroelec., Freq. Contr., vol. 61, no. 2, pp. 288-301, Feb 2014.

[168] L. Tong, R. A., P. Tortoli, G. Fradella, S. Caciolli, J. Luo, and J. D'Hooge, "Wide-angle tissue doppler imaging at high frame rate using multi-line transmit beamforming: an experimental validation invivo," IEEE Trans. Med. Imag., vol. PP, no. 99, p. In Press, 2016.

[169] K. L. Hansen, H. Møller-Sørensen, J. Kjaergaard, M. B. Jensen, J. T. Lund, M. M. Pedersen, T. Lange, J. A. Jensen, and M. B. Nielsen, "Flow in the ascending aorta in patients with normal, stenotic and replaced aortic valves - a study with transverse oscillation," Ultrasound Med. Biol., p. Submitted, 2016.

[170] K. L. Hansen, M. M. Pedersen, H. Møller-Sørensen, J. Kjaergaard, J. C. Nilsson, J. T. Lund, J. A. Jensen, and M. B. Nielsen, "Intraoperative 
cardiac ultrasound examination using vector flow imaging," Ultrason. Imaging, vol. 35, no. 4, pp. 318-332, Oct 2013.

[171] N. L. Greenberg, P. M. Vandervoort, M. S. Firstenberg, M. J. Garcia, and J. D. Thomas, "Estimation of diastolic intraventricular pressure gradients by Doppler M-mode echocardiography," Am. J. Physiol. Heart Circ. Physiol., vol. 280, no. 6, pp. H2507-H2515, 2001.

[172] R. Yotti, J. Bermejo, M. M. Desco, J. C. Antoranz, J. L. Rojo-Alvarez, C. Cortina, C. Allua, H. Rodraguez-Abella, M. Moreno, and M. A. Garcia-Fernanndez, "Doppler-derived ejection intraventricular pressure gradients provide a reliable assessment of left ventricular systolic chamber function," Circulation, vol. 112, no. 12, pp. 1771-1779, 2005, pMID: 16172285.

[173] J. C. del Alamo, D. Garcia, D. Tanne, C. Cortina, R. Yotti, F. Fernandez-Aviles, and J. Bermejo, "Two-dimensional intraventricular mapping of pressure and vorticity by digital processing conventional color-Doppler sequences," J. Amer. Coll. Cardio., vol. 53, no. 10, pp. A375-A375, March 2009.

[174] J. B. Olesen, M. S. Traberg, M. J. Pihl, and J. A. Jensen, "Noninvasive esimation of 2-D pressure gradients in steady flow using ultrasound," IEEE Trans. Ultrason., Ferroelec., Freq. Contr., vol. 61, no. 8, pp. 1409-1418, 2014.

[175] J. B. Olesen, C. A. Villagomez-Hoyos, M. S. Traberg, and J. A. Jensen, "Non-invasive estimation of pressure gradients in pulsatile flow using ultrasound," in Proc. IEEE Ultrason. Symp. IEEE, 2014, pp. 22572260 .

[176] C. J. Slager, J. J. Wentzel, F. J. H. Gijsen, J. C. H. Schuurbiers, A. van der Wal, A. F. W. van der Steen, and P. W. Serruys, "The role of shear stress in the generation of rupture-prone vulnerable plaques," Nature Reviews Cardiology, vol. 2, no. 8, pp. 401-407, 2005.

[177] K. L. Hansen, H. Møller-Sørensen, J. Kjaergaard, J. T. Lund, M. M. Pedersen, T. Lange, J. A. Jensen, and M. B. Nielsen, "Analysis of systolic backflow and secondary helical blood flow in the ascending aorta using vector flow imaging," Ultrasound Med. Biol., p. In press, 2016.

[178] M. D. Hope, T. A. Hope, A. K. Meadows, K. G. Ordovas, T. H Urbania, M. T. Alley, and C. B. Higgins, "Bicuspid aortic valve: fourdimensional MR evaluation of ascending aortic systolic flow patterns," Radiology, vol. 255, no. 1, pp. 53-61, 2010.

[179] A. Swillens, J. Degroote, J. Vierendeels, L. Løvstakken, and P. Segers, "A simulation environment for validating ultrasonic blood flow and vessel wall imaging based on fluid-structure interaction simulations: Ultrasonic assessment of arterial distension and wall shear rate," Medical Physics, vol. 37, no. 8, pp. 4318-4330, Aug 2010.

[180] M. Qian, L. Niu, K. Wong, D. Abbott, Q. Zhou, and H. Zheng, "Pulsatile flow characterization in a vessel phantom with elastic wall using ultrasonic particle image velocimetry technique: the impact of vessel stiffness on flow dynamics," IEEE Trans. Biomed. Eng., vol. 61, no. 9, pp. 2444-2450, 2014.

[181] A. Arbab-Zadeh and V. Fuster, "The myth of the 'vulnerable plaque': transitioning from a focus on individual lesions to atherosclerotic disease burden for coronary artery disease risk assessment," Journal of the American College of Cardiology, vol. 65, no. 8, pp. 846-855, 2015.

[182] A. H. Brandt, K. L. Hansen, M. B. Nielsen, and J. A. Jensen, "Velocity estimation of the main portal vein with transverse oscillation." in Proc. IEEE Ultrason. Symp. IEEE, 2015, pp. 1-4.

[183] R. S. Laramee, H. Hauser, H. Doleisch, B. Vrolijk, F. H. Post, and D. Weiskopf, "The state of the art in flow visualization: Dense and texture-based techniques," Computer Graphics Forum, vol. 23, no. 2, pp. 203-221, 2004.

\section{BIBLIOGRAPHIES}

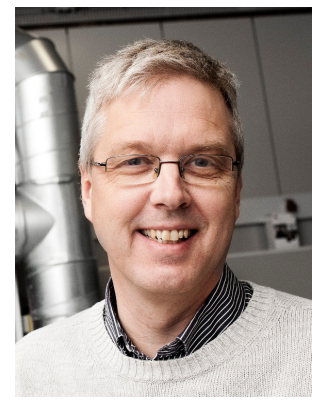

Jørgen Arendt Jensen earned his Master of Science in electrical engineering in 1985 and the $\mathrm{PhD}$ degree in 1989, both from the Technical University of Denmark. He received the Dr.Techn. degree from the university in 1996. He has since 1993 been full professor of Biomedical Signal Processing at the Technical University of Denmark at the Department of Electrical Engineering and head of Center for Fast Ultrasound Imaging since its inauguration in 1998. He has published more than 450 journal and conference papers on signal processing and medical ultrasound and the book "Estimation of Blood Velocities Using Ultrasound", Cambridge University Press in 1996. He is also the developer and maintainer of the Field II simulation program. He has been a visiting scientist at Duke University, Stanford University, and the University of Illinois at Urbana-Champaign. He was head of the Biomedical Engineering group from 2007 to 2010. In 2003 he was one of the founders of the biomedical engineering program in Medicine and Technology, which is a joint degree program between the Technical University of Denmark and the Faculty of Health and Medical Sciences at the University of Copenhagen. The degree is one of the most sought after engineering degrees in Denmark. He was chairman of the study board from 2003-2010 and adjunct professor at the University of Copenhagen from 2005-2010. He has given a number of short courses on simulation, synthetic aperture imaging, and flow estimation at international scientific conferences and teaches biomedical signal processing and medical imaging at the Technical University of Denmark. He has given more than 60 invited talks at international meetings, received several awards for his research, and is an IEEE Fellow. His research is centered around simulation of ultrasound imaging, synthetic aperture imaging, vector blood flow estimation, and construction of ultrasound research systems.

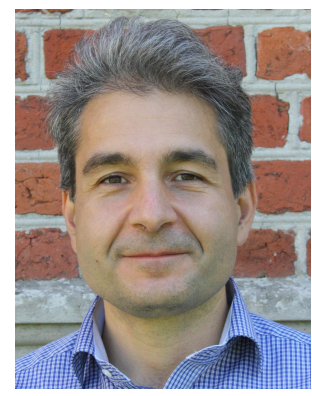

Svetoslav Ivanov Nikolov got his Master of Science in electrical engineering in 1996 from the Technical University - Sofia (TU - Sofia), and a Ph.D. degree in 2001 fromt the Technical University of Denmark 
(DTU). From 2001 till 2009 he was an Associate Professor in Electronics and Signal Processing at DTU. Svetoslav Nikolov is Senior IEEE Member since 2011 for his pioneering work in the field of synthetic aperture imaging and flow estimation. In 2008, he joined BK Medical Aps to work on the commercial implementation of the technologies developed at DTU. He is an Analogic Fellow since 2015 as a recognition for his work in research and development.

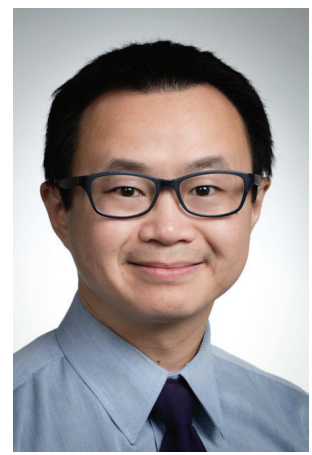

Alfred C. H. Yu (S99-M07-SM12) is an Associate Professor in the Department of Electrical and Computer Engineering at the University of Waterloo, ON, Canada. He has a long-standing research interest in ultrasound imaging innovations and therapeutic ultrasound discoveries. Alfred obtained his undergraduate degree in Electrical Engineering from the University of Calgary in 2002, and he received his M.A.Sc. and Ph.D. degrees in Biomedical Engineering from the University of Toronto in 2004 and 2007. He also interned at Philips Research North America in 2005. Before he relocated to Waterloo, Alfred was a Research Assistant Professor at the University of Hong Kong, where he founded and served as the Principal Investigator of HKU Biomedical Ultrasound Laboratory. Alfred is a Senior Member of IEEE and AIUM. He is an Associate Editor of IEEE Transactions on Ultrasonics, Ferroelectrics, and Frequency Control and an Editorial Board Member of Ultrasound in Medicine and Biology. He also serves on the Technical Program Committee of IEEE Ultrasonics Symposium and International Symposium for Therapeutic Ultrasound.

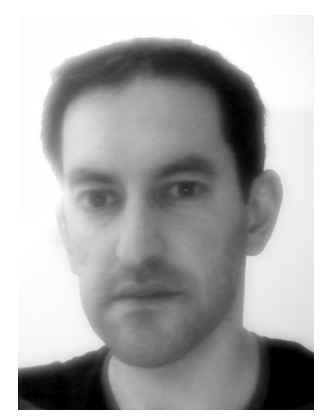

Damien Garcia obtained his engineer degree in Mechanical Engineering from the cole Centrale de Marseille, France, in 1997, and received his Ph.D. degree in Biomedical Engineering from the University of Montreal, Canada, in 2003. He was a postdoctoral fellow from 2006 to 2008 in the Department of Echocardiography,
Gregorio Marañón hospital, Madrid, Spain. He is director of the Research Unit of Biomechanics \& Imaging in Cardiology (RUBIC) at the University of Montreal Hospital Research Centre (CRCHUM), and assistant professor at the Department of Radiology, Radio-Oncology and Nuclear Medicine at the University of Montreal. He is an Associate Editor of IEEE Transactions on Ultrasonics, Ferroelectrics, and Frequency Control and is on the Technical Program Committee of the IEEE Ultrasonics Symposium. His clinical and fundamental research interests are in cardiac/cardiovascular ultrasound imaging, mostly in fluid dynamics and flow imaging. A detailed list of his publications is available at www.biomecardio.com 